\title{
Numerical simulation of tribological devices used as a set of benchmarks for comparing contact algorithms
}

\author{
P. Chabrand ${ }^{\mathrm{a}}$, F. Dubois ${ }^{\mathrm{b}}$, D. Graillet ${ }^{\mathrm{c}}$, R. Boman ${ }^{\mathrm{c}}$, J.P. Ponthot $^{\mathrm{c}, *}$ \\ ${ }^{a}$ Laboratoire de Mécanique et d'Acoustique - UPR CNRS 7051, 31, Chemin Joseph Aiguier 13402 Marseille Cedex 20, France \\ ${ }^{\mathrm{b}}$ Laboratoire de Mécanique et Génie Civil - UMR CNRS 5508, Université de Montpellier II, Place Eugène Bataillon, \\ 34095 Montpellier Cedex 05, France \\ ${ }^{\mathrm{c}}$ L.T.A.S - Milieux Continus et Thermomécanique, Université de Liège, 1 chemin des chevreuils, B4000 Liège, Belgium
}

The aim of the present study was to carry out a numerical comparison of different frictional contact algorithms. Therefore three different contact algorithms (Lemke, penalty and Augmented Lagrangian) have been implemented into two finite element codes. The correct implementation and behavior of these contact algorithms has been investigated by modeling four different tribological devices. It is shown that all these different methodologies lead to extremely similar results. Besides these four applications have been carefully described and detailed in such a way that the presented tests can be reproduced. The authors wish that they could serve as a benchmark set in order to allow comparison with other finite element software including frictional contact capabilities.

Keywords: Friction; Numerical; Simulations; Drawbead; Deep drawing; Finite element

\section{Introduction}

Friction is known to be one of the main factors involved in many forming processes. For example in deep drawing processes, restraining forces are set up between sheet and forming tools by friction, as well as by the bending/unbending of the sheet. The friction coefficient depends on the surface roughness, the presence or absence of a lubricant, the clamping pressure and the sliding velocity. Experimental measurements are

\footnotetext{
* Corresponding author. Tel.: +3243669310; fax: +3243669141.

E-mail address: jp.ponthot@ulg.ac.be (J.P. Ponthot).
} 
used to obtain a global friction coefficient, which is commonly used for local scale simulations [1] . In some studies, variable friction coefficients have been determined to fit Stribeck curves or local conditions $[2,3]$. As a matter of fact plastic deformations of the surface asperities, lubricant temperature and viscosity are, for example, local physical parameters which strongly influence the friction coefficient value.

In most cases the information obtained from experiments are global quantities and from these data it is very difficult to extract information on the local level (local pressure, contact force distribution, true contact area, etc). On the other hand, the numerical simulation allows to retrieve local information and to provide, associated with the experimental analysis, a powerful tool aimed at a better understanding of the contact physics. Numerical treatment of contact problems arising from modeling tribological devices is complex due to the strong nonlinearities involved, see e.g., [4]. These nonlinearities arise from the complex material behavior, from large changes in geometry and from the frictional contact itself. Therefore, a lot of care has to be dedicated to the verification of the algorithms implementation.

The key point of the present work is to cross validate frictional algorithms by comparing results obtained with various algorithms implemented in two different finite element code. To achieve this goal, we have chosen four tests which include most of the particularities found in experimental devices. The presentation of these tests is given in detail to allow future reproduction of these simulations.

In the present study, which deals with the use of a constant global friction coefficient, we investigate the effects of the material behavior and the tool and the sheet geometry on the friction forces. The first problem studied is a classical flat die simulator in which the contact forces are distributed along the contact surfaces. With our models it is possible to deal with rigid tools and to prescribe constant clamping forces as well as constant tool displacements $[5,6]$.

The effects of bending/unbending depend strongly on the sheet thickness and the tool radius. Numerical simulation can provide useful information about the local tool contact conditions. It is then possible to determine the contact force intensity and the distribution of the contact states which cannot be determined experimentally. A radial strip drawing test was used to illustrate this point. From the numerical point of view, our aim was to study with this test the effects of the mesh and those of the sheet thickness/tool radius ratio and the wrapping angle on the contact state.

In industrial deep drawing processes, drawbeads play a very important role. However, due to complex combination of bending/unbending strains and friction forces acting on the blank what actually happens in the drawbead is not clearly understood. In order to better understand the involved phenomena, Nine [7-9] designed an experimental drawbead simulation rig which allows to separate the contribution of the bending forces from the contribution of the friction forces. Numerical simulation of this experimental device is the object of our third application.

Lastly, our fourth example consists in modeling a blank-holder fitted with a drawbead. The effects of the blank-holder and die geometry are discussed as well as those resulting from the drawbead. The restraining forces are determined in each case.

These tests were modeled numerically assuming an elasto-plastic behavior in the context of finite strains. An isotropic hardening law is used in the formulation. Based on an hyper-elastic regularization, a mixed formulation can be derived which gives a good description of the plastic incompressibility, even in the case of elements with a low degree of interpolation. The contact was assumed to occur between a deformable body (the workpiece) and a rigid obstacle (the tool). In the case of a frictional multibody contact problem, a slave/master approach was used [10]. The models were implemented in two different finite element codes: SIMEM3 at the LMA in Marseille and METAFOR at the LTAS in Liège for comparison purposes. 
From the numerical point of view, various methods dealing with the treatment of frictional contact problems were studied and compared with our finite element codes. At the LMA, a Lemke's method was used with which the frictional contact problem can be exactly solved without requiring any additional parameters or approximation [11]. On the other hand, METAFOR uses penalty and Augmented Lagrangian methods to solve the contact problem: these efficient methods were developed by appropriately regularizing the impenetrability and friction conditions.

The paper is organized as follows. We will begin by describing the finite elasto-plastic formulation. We then briefly describe the numerical methods including flow charts, developed to deal with the frictional contact problem in the two finite element code. The last part deals with the simulation of the four experimental devices. Geometry of the tools and material properties are described extensively. The numerical results are compared and discussed in details.

\section{Equilibrium equations}

In the displacement based finite element method, the discretized form of the equilibrium equations is used to calculate the estimated incremental displacement. A modified Newton-Raphson method is used to deal with the non-linear equations arising from the finite deformations, the constitutive equations and the frictional contact conditions. The loading consists of a sequence of steps, at each of which an iterative process is performed to solve the set of non-linear equations written on successive intermediate configurations. We denote $\Omega_{n}$ the reference configuration at the end of loading step $n$. Configuration $\Omega_{n+1}$ is computed iteratively from $\Omega_{n}$ (which is known from the previous loading step) by performing iterations $i$ of the Newton-Raphson method with the corresponding intermediate configurations $\Omega_{n+1}^{i}$.

Let $u_{n}$ be the nodal displacement vector at the end of loading step $n$. We denote $\Delta u_{n+1}^{i}$ the incremental displacement between configurations $\Omega_{n}$ and $\Omega_{n+1}^{i}$ and $d u^{i+1}$ the displacement between $\Omega_{n+1}^{i}$ and $\Omega_{n+1}^{i+1}$. Hence

$$
\Delta u_{n+1}^{i+1}=\Delta u_{n+1}^{i}+\mathrm{d} u^{i+1}, \quad u_{n+1}^{i+1}=u_{n}+\Delta u_{n+1}^{i+1} .
$$

The equilibrium equation of the configuration $\Omega_{n+1}^{i}$ is:

$$
\left\{\operatorname{Res}^{i}\right\}=\left\{F^{\mathrm{int}}\left(u_{n+1}\right)^{i}\right\}-\left\{F_{n+1}^{\mathrm{ext}}\right\}-\left\{R^{i}\right\}=0,
$$

where $\left\{\operatorname{Res}^{i}\right\}$ denotes the equilibrium residual vector obtained by assembling the internal and external forces. $\left\{F^{\text {int }}\left(u_{n+1}\right)^{i}\right\}$ is the discrete load vector corresponding to the internal stresses, and $\left\{F_{n+1}^{\text {ext }}\right\}$ is the discrete load vector corresponding to the external forces excluding the contact forces. $\left\{R^{i}\right\}$ is the unknown vector of the contact forces. Using Newton's method for generalized equations (Klarbring et al. [12]), a linearized form of (2) can be derived

$$
\left[K_{\mathrm{T}}\right]\{\mathrm{d} u\}^{i+1}=-\left\{F^{\mathrm{int}}\left(u_{n+1}\right)^{i}\right\}+\left\{F_{n+1}^{\mathrm{ext}}\right\}+\left\{R^{i+1}\right\}
$$

where $K_{\mathrm{T}}$ is the tangent stiffness matrix consistent with constitutive equation integration and contact force calculations. 


\section{Material behavior}

In the present study, large deformations have to be taken into account. The material is assumed to have an isotropic elasto-plastic behavior involving isotropic hardening. The model adopted is characterized by an intermediate configuration. It leads to the local multiplicative decomposition of the deformation gradient $F$ into its elastic $F^{\mathrm{e}}$ and plastic $F^{\mathrm{p}}$ counterparts, as proposed by Lee [13]

$$
F=F^{\mathrm{e}} F^{\mathrm{p}} \text {. }
$$

The constitutive laws of the model are determined by introducing the free energy function

$$
\Psi\left(b^{\mathrm{e}}, \bar{\varepsilon}^{\mathrm{p}}\right)=\Psi^{\mathrm{e}}\left(b^{\mathrm{e}}\right)+\Psi^{\mathrm{p}}\left(\bar{\varepsilon}^{\mathrm{p}}\right),
$$

where the elastic potential $\Psi^{\mathrm{e}}$ is an isotropic function of the left Cauchy Green strain tensor

$$
b^{\mathrm{e}}=F^{\mathrm{e}} F^{\mathrm{e}^{\mathrm{T}}}
$$

and where $\bar{\varepsilon}^{\mathrm{p}}$ denotes the isotropic hardening variable (equivalent plastic strain) and $\Psi^{\mathrm{p}}$ the hardening potential free energy.

The behavior between the intermediate and current configurations is given by a hyper-elastic response. The Kirchhoff stress tensor is then given by the classical constitutive equation [14]

$$
\tau=2 b^{\mathrm{e}} \frac{\partial \Psi^{\mathrm{e}}}{\partial b^{\mathrm{e}}} .
$$

The intermediate configuration is up-dated by integrating plastic evolutionary laws. The plastic response of the material is described using the classical Von-Mises yield condition. The associative flow rule determined by the principle of maximum plastic dissipation is written as

$$
d^{\mathrm{p}}=\lambda \sqrt{\frac{3}{2}} \frac{\operatorname{dev}(\tau)}{\|\operatorname{dev}(\tau)\|},
$$

where $d^{\mathrm{p}}$ is the plastic strain rate, $\lambda$ is the plastic multiplier and $\operatorname{dev}(\tau)$ denotes the deviatoric part of the Kirchhoff stress. The Von-Mises yield function associated to (8) is defined as:

$$
\sqrt{\frac{3}{2}}\|\operatorname{dev}(\tau)\|-\sigma_{\mathrm{v}}\left(\bar{\varepsilon}^{\mathrm{p}}\right)=0,
$$

where $\bar{\varepsilon}^{\mathrm{p}}=\int_{0}^{t} \sqrt{\frac{2}{3} d \mathrm{p}: d^{\mathrm{p}}} \mathrm{d} t$ is the equivalent plastic strain and $\sigma_{\mathrm{v}}\left(\bar{\varepsilon}^{\mathrm{p}}\right)$ the isotropic hardening law.

From the computational point of view, the above constitutive equations can be integrated using an elastic predictor/plastic corrector algorithm. We assume that at time $t_{n}$ with the configuration $\Omega_{n}$ of the body, the state variables $\left(F_{n}^{\mathrm{e}}, F_{n}^{\mathrm{p}}, \tau_{n}\right)$ are known. Given $\Delta u_{n+1}$ the displacement between the configurations $\Omega_{n}$ and $\Omega_{n+1}$, we can perform an elastic prediction, assuming that the plastic variables remain frozen $\left(F_{n+1}^{\mathrm{p}}=F_{n}^{\mathrm{p}}\right)$. The trial elastic part of the deformation gradient (4) as well as the trial elastic Kirchhoff stress (7) can be then computed. If the stress lies outside the elastic region, a plastic correction step is then carried out using a classical radial return algorithm [15]. Alternatively, a hypo-elastic algorithm can be used without affecting significantly the results, see [16] for details. 


\section{Contact and friction treatment}

\subsection{Introduction}

The interface behavior is described in terms of unilateral contact conditions involving Coulomb's friction. Let $n$ be the outward normal unit vector to the rigid tool. We use the following decomposition into the normal and tangential components of the displacements $u$ and the contact force vector $R$ :

$$
\begin{aligned}
& u_{\mathrm{N}}=u . n, \quad u_{\mathrm{T}}=u-u_{\mathrm{N}} n, \\
& R=R_{\mathrm{N}} n+R_{\mathrm{T}} .
\end{aligned}
$$

Let $g_{\mathrm{N}}^{0}$ be the gap function at the start of the loading step. The unilateral contact conditions can then be written in terms of the relative displacements $\Delta u$ as follows:

$$
\begin{aligned}
& g_{\mathrm{N}}=\Delta u_{\mathrm{N}}+g_{\mathrm{N}}^{0}, \\
& g_{\mathrm{N}} \geqslant 0, \quad R_{\mathrm{N}} \geqslant 0, \quad g_{\mathrm{N}} \cdot R_{\mathrm{N}}=0 .
\end{aligned}
$$

As a friction condition for the tangential direction, we take Coulomb's friction law with constant coefficient, written below in an incremental form, in which $g_{\mathrm{T}}^{0}$ denotes the tangential incremental displacement of the rigid body and $g_{\mathrm{T}}$ the relative tangential displacement:

$$
\begin{aligned}
& g_{\mathrm{T}}=\Delta u_{\mathrm{T}}-g_{\mathrm{T}}^{0} \\
& \text { with: }\left\|R_{\mathrm{T}}\right\| \leqslant \mu R_{\mathrm{N}}, \quad\left\{\begin{array}{l}
\left\|R_{\mathrm{T}}\right\|<\mu R_{\mathrm{N}} \Rightarrow g_{\mathrm{T}}=0 \\
\left\|R_{\mathrm{T}}\right\|=\mu R_{\mathrm{N}} \Rightarrow g_{\mathrm{T}}=-\alpha R_{\mathrm{T}}, \alpha \geqslant 0 .
\end{array}\right.
\end{aligned}
$$

\subsection{Complementarity approach}

As a means of dealing with the three-dimensional case, Klarbring et al. [12] have introduced a piecewise linear approximating Coulomb's friction law. This discretization procedure makes it possible to write the friction relations as complementarity conditions and then to set the problem as a linear complementarity one. In the present study, we shall restrict ourselves to a two-dimensional analysis. In this situation, the approximation of Coulomb's law leads to introducing two new variables, $\lambda$ and $\phi$, which define the boundary of the Coulomb's cone. The Kuhn Tucker conditions for the frictional Coulomb problem can then be written in the following form:

$$
\begin{aligned}
& R_{\mathrm{T}} \in C\left(R_{\mathrm{N}}\right), \\
& \phi_{1}\left(R_{\mathrm{T}}, R_{\mathrm{N}}\right)=-R_{\mathrm{T}}+\mu R_{\mathrm{N}}, \\
& \phi_{2}\left(R_{\mathrm{T}}, R_{\mathrm{N}}\right)=R_{\mathrm{T}}+\mu R_{\mathrm{N}}, \\
& g_{\mathrm{T}}=-\lambda_{1} \frac{\partial \phi_{1}}{\partial R_{\mathrm{T}}}-\lambda_{2} \frac{\partial \phi_{2}}{\partial R_{\mathrm{T}}}, \\
& \lambda_{m} \geqslant 0, \quad \phi_{m} \geqslant 0, \quad \lambda_{m} \phi_{m}=0 \quad(m=1,2)
\end{aligned}
$$

with $C\left(R_{\mathrm{N}}\right)=\left\{P_{\mathrm{T}}, \phi_{m}\left(P_{\mathrm{T}}, R_{\mathrm{N}}\right) \geqslant 0, m=1,2\right\}$. 
Expressing the initial system (3) in terms of local $(n, t)$ components instead of global $(x, y)$ one's, we can reformulate the systems in terms of unknowns concerned by contact or friction $(c)$ and free components $(f)$ :

$$
\left[\begin{array}{ll}
K_{T_{c c}} & K_{T_{c f}} \\
K_{T_{f c}} & K_{T_{f f}}
\end{array}\right]\left[\begin{array}{l}
\mathrm{d} u_{c} \\
\mathrm{~d} u_{f}
\end{array}\right]=\left[\begin{array}{c}
F_{c} \\
F_{f}
\end{array}\right]+\left[\begin{array}{c}
R_{c} \\
0
\end{array}\right]
$$

with $F=F^{\text {ext }}-F^{\text {int }}$.

Thanks to a condensation procedure, system (14) can be written as two connected ones:

$$
\begin{aligned}
& K^{*} \mathrm{~d} u_{c}=F^{*}+R_{c}, \\
& \mathrm{~d} u_{f}=K_{T_{f f}}^{-1} F_{f}-K_{T_{f f}}^{-1} K_{T_{f c}} \mathrm{~d} u_{c},
\end{aligned}
$$

with

$$
\begin{aligned}
& K^{*}=K_{T_{c c}}-K_{T_{c f}} K_{T_{f f}}^{-1} K_{T_{f c}}, \\
& F^{*}=F_{c}-K_{T_{c f}} K_{T_{f f}}^{-1} F_{f} .
\end{aligned}
$$

The first system deals only with the components of contact nodes. Let $n c$ be the number of contact nodes it is a square $2 n c * 2 n c$ system. Upon doing some changes of variables and introducing variables $\lambda$ and $\phi$, the complementarity pairs of variables associated with each contact node become: $\left(\Delta w_{\mathrm{N}}, R_{\mathrm{N}}\right)$ and $\left(\lambda_{i}, \phi_{i} i=1,2\right)$. All the components of this system are then constrained by complementarity conditions. Each contact nodes is related to 3 pairs of complementarity unknowns instead of 2 . This linear complementarity problem can then be straightforwardly solved using a pivot algorithm such as Lemke's method [11].

The second system deals with the nodes which are not involved in the contact. This is a non constrained problem in which the only unknown vector is the displacement one. Its solution obviously depends on the solution of the previous system, and can be obtained by classically solving a linear system.

\subsection{Penalty/Augmented Lagrangian}

METAFOR [17] instead of solving the exact analytical contact with friction conditions such as done by Lemke's method uses a well-known regularization method called the penalty method [18]. In this method, the nodal reactions, resulting from contact between a contact node and a contact surface, are directly proportional to the penetration (orthogonal component) and, in case of sticking contact, the tangential displacements (tangential components) along the contact surface encountered. Since this method is a regularization method based on the penalty principle, it does not exactly fulfill the conditions of impenetrability and perfect sticking: the violation of these two conditions is therefore penalized through the penalty coefficients. 
In fact, the penalty method needs some nonzero penetration to generate a normal contact force (one can physically describe this penetration in terms of an elastic deformation of the contact surface), as well as some tangential motion to generate the tangential forces, even if the contact is sticking (here again, this might correspond to some elastic shear deformation of the asperities produced by the tangential forces).

From the practical point of view, the penalty coefficients have to be large enough to reduce these violations to a very small proportion of the total deformation. This means that the contact will not involve penetrations which are too large or sticking points which are too far from the point where they should be to exactly fulfill the non-sliding conditions.

The penalty method yields for contact with Coulomb friction [18]:

$$
\begin{aligned}
& R_{\mathrm{N}}=\left\langle-\varepsilon_{\mathrm{N}} g_{\mathrm{N}}\right\rangle \\
& R_{\mathrm{T}}=R_{\mathrm{T}}^{\mathrm{pr}}-\left\langle-\Psi^{s l}\right\rangle \operatorname{sign}\left(R_{\mathrm{T}}^{\mathrm{pr}}\right) \\
& R_{\mathrm{T}}^{\mathrm{pr}}=-\varepsilon_{\mathrm{T}} g_{\mathrm{T}} ; \quad \Psi^{s l}=\mu\left\|R_{\mathrm{N}}\right\|-\left\|R_{\mathrm{T}}^{\mathrm{pr}}\right\|,
\end{aligned}
$$

where $R_{i}$ are the contact forces, $\varepsilon_{i}$ the penalty coefficients and $g_{i}$ the gaps (computed in the local axis) and where $\langle x\rangle$ denotes the Mac Auley brackets, i.e., $\langle x\rangle=(x+|x|) / 2$. With the subscripts $\mathrm{N}_{\mathrm{N}}$ and $\mathrm{T}_{\mathrm{T}}$ for respectively the orthogonal and tangential counterparts, $R_{\mathrm{T}}^{\mathrm{pr}}$ is the sticking predictor of the tangential contact force and the sliding corrector $\Psi^{s l}$ ensures that this force will not exceed the maximal value fixed by the Coulomb Law (i.e., $\mu\left\|R_{\mathrm{N}}\right\|$ ). All these quantities are evaluated in local axis which will evolve with the deformation or even with the position if the contact surfaces exhibit some curvature. In such a nonlinear geometrical framework, attention has to be paid to a correct treatment that meets the frame invariance or objectivity requirement. Details of the appropriate treatment to correctly deal with this can be found in the textbook by Laursen [18].

Another regularization method the Augmented Lagrangian method [19], is also available in METAFOR. This method can be easily compared to the penalty method since one can write:

$$
\begin{aligned}
& R_{\mathrm{N}}=\left\langle\lambda_{\mathrm{N}}-\varepsilon_{\mathrm{N}} g_{\mathrm{N}}\right\rangle \\
& R_{\mathrm{T}}=R_{\mathrm{T}}^{\mathrm{pr}}-\left\langle-\Psi^{s l}\right\rangle \operatorname{sign}\left(R_{\mathrm{T}}^{\mathrm{pr}}\right) \\
& R_{\mathrm{T}}^{\mathrm{pr}}=\lambda_{\mathrm{T}}-\varepsilon_{\mathrm{T}} g_{\mathrm{T}} ; \quad \Psi^{s l}=\mu\left\|R_{\mathrm{N}}\right\|-\left\|R_{\mathrm{T}}^{\mathrm{pr}}\right\|
\end{aligned}
$$

in which the pseudo-Lagrangian coefficients $\lambda_{\mathrm{N}}$ and $\lambda_{\mathrm{T}}$ are introduced. These terms are not additional unknowns, as in traditional Lagrangian methods where $\lambda_{\mathrm{T}}$ and $\lambda_{\mathrm{N}}$ are to be determined in the Newton process, therefore increasing the size of the linear system to be solved, but are iteratively set to the approximation of the surface traction obtained during the convergence process, see $[19,20,18,5,6]$ for details.

\section{Force driven blankholder}

In the forming of sheet metal parts, the quality of the final products, in terms of the absence of wrinkles and necking, depends on the restraining forces exerted by the blank-holder on the sheet. 
In the case of rigid tools which are not discretized in the finite element sense, the normal load is characterized by the displacement of the center of gravity of the tool. To account for the clamping force two ways are then possible. The tool can be displacement or force driven. In the first case the solution is trivial since the tool position is described as an explicit function of time. Therefore at a given time the tool position can be simply determined by evaluating a known function. Due to the material deformation associated with the drawing the clamping forces resulting from the tool imposed displacement is an evolving quantity that changes with time even if the tool position is fixed. In the force driven case it is the clamping force that is an explicit function of time. As a consequence it is then necessary to adapt the tool position to satisfy the prescribed applied force at the center of gravity. In a such situation, the position of the tool becomes an additional unknown of the problems which has to be determined at each increment while solving the whole problem. We present below the algorithms used in conjunction with the different contact algorithms.

\subsection{LMA treatment}

The blank-holder is taken to be a moving body, the (vertical) position of which is determined in order to exactly equilibrate the (vertical) loads acting on it by the jack pressure and the reactions due to the frictional contact with the sheet. Introducing this new degree of freedom ( $\Delta \lambda$ assumed to be vertical $(y))$ leads, in the case of a node in contact with the blank-holder, to the following parametric form of the normal and tangential relative displacements:

$$
\begin{aligned}
& g_{\mathrm{N}}=\Delta u_{\mathrm{N}}+g_{\mathrm{N}}^{0}-\Delta \lambda n_{y}, \\
& g_{\mathrm{T}}=\Delta u_{\mathrm{T}}+g_{\mathrm{T}}^{0}-\Delta \lambda t_{y} .
\end{aligned}
$$

Rewriting system (15) in terms of components in contact with the blank-holder ( $b, n e q_{b}$ components) and others $(o)$ :

$$
\left[\begin{array}{ll}
K_{b b} & K_{b o} \\
K_{o b} & K_{o o}
\end{array}\right]\left[\begin{array}{l}
g_{b} \\
g_{o}
\end{array}\right]=\left[\begin{array}{l}
F_{b} \\
F_{o}
\end{array}\right]+\left[\begin{array}{l}
R_{b} \\
R_{o}
\end{array}\right] .
$$

We introduce into our system the new unknown $\Delta \lambda$ and a new equation expressing the equilibrium of the blank-holder: the sum of the vertical contact reactions (due to contact and friction) equals the jack pressure $\left(F_{b l h}\right)$. After some intermediate calculus (Dubois [5]), we obtain the following formal system:

$$
\left[\begin{array}{lll}
K_{b b} & K_{b o} & K_{b \lambda} \\
K_{o b} & K_{o o} & K_{o \lambda} \\
K_{\lambda b} & K_{\lambda b} & K_{\lambda \lambda}
\end{array}\right]\left[\begin{array}{l}
g_{b} \\
g_{o} \\
\Delta \lambda
\end{array}\right]=\left[\begin{array}{l}
F_{b} \\
F_{o} \\
F_{\lambda}
\end{array}\right]+\left[\begin{array}{l}
R_{b} \\
R_{o} \\
R_{\lambda}
\end{array}\right],
$$

where

$$
\begin{aligned}
& K_{b_{i} \lambda}=\sum_{j=1}^{n e q_{b}} K_{b_{i} b_{j}} k_{j}, \quad K_{o_{i} \lambda}=\sum_{j=1}^{n e q_{b}} K_{o_{i} b_{j}} k_{j}, \\
& K_{\lambda b_{j}}=\sum_{i=1}^{n e q_{b}} k_{i} K_{b_{i} b_{j}}, \quad K_{\lambda o_{j}}=\sum_{i=1}^{n e q_{b}} k_{i} K_{o_{i} b_{j}},
\end{aligned}
$$




$$
\begin{aligned}
& K_{\lambda \lambda}=\sum_{i=1}^{n e q_{b}} k_{i} K_{b_{i} \lambda}, \\
& R_{\lambda}=\sum_{i=1}^{n e q_{b}} k_{i} P_{b_{i}}, \quad F_{\lambda}=\sum_{i=1}^{n e q_{b}} k_{i} F_{b_{i}}, \\
& k_{j}= \begin{cases}n_{y_{i b}} & \text { if } j \text { relates to the contact } \\
t_{y_{i b}} & \text { if } j \text { relates to the friction } \\
& \text { component of the node } i b .\end{cases}
\end{aligned}
$$

In order to enforce $R_{\lambda}=F_{b l h}$, we use Lemke's method both with the following obvious change of variable:

$$
\begin{aligned}
& \Delta \tilde{\lambda}=\Delta \lambda+\alpha \quad \text { with } \alpha \gg 0, \\
& \tilde{R}_{\lambda}=R_{\lambda}-F_{\text {blh }} .
\end{aligned}
$$

The couple $\left(\Delta \tilde{\lambda}, \tilde{R}_{\lambda}\right)$ must satisfy complementary conditions, due to the former change of variable $\Delta \tilde{\lambda}>0$ and $\tilde{R}_{\lambda}=0$.

\subsection{LTAS treatment}

For each rigid tool which has to be force driven, new degrees of freedom (d.o.f.) are introduced in the finite element solution procedure. If a tool is force driven in only one direction, as in most practical cases, only one degree of freedom is introduced (for a traditional blank-holder, the clamping direction needs a new d.o.f. and the perpendicular direction is fixed). In METAFOR, contact elements (nodes of the boundary of the deformable body) are associated to a rigid tool. The displacement of these nodes have to be coupled to the displacement of the tool, so that the resulting contact force equals the desired one. Each new d.o.f. of the tool and the nodes in contact lead to the definition of a "force" finite element, for which an elementary external and internal force vector $\left(F_{M}^{\text {ext }}\right.$ and $\left.F_{M}^{\text {int }}\right)$ and a stiffness matrix $\left(K_{\mathrm{T}}\right)$ can be derived.

As described in Section 2, the equilibrium equations are solved iteratively with a Newton-Raphson procedure. If we restrict ourselves to an implicit and quasistatic computation, the equilibrium equation associated to a new d.o.f. of a force driven tool can be written

$$
F_{M}^{\mathrm{int}}=F_{M}^{\mathrm{ext}}
$$

This equation is added to the equilibrium equations of the deformable body and the linearization of the whole system defines the tangent stiffness matrix $\left(K_{\mathrm{T}}\right)$ associated to the "force" element. The size of this 
matrix equals to

$$
\operatorname{dim}\left(K_{\mathrm{T}}\right)=n e q_{b}+I=n c_{b} * n o d d l+I,
$$

where noddl is the number of d.o.f. by contact node ( 2 in $2 \mathrm{D}), n c_{b}$ is the number of nodes currently in contact with the blankholder, $I$ is the number of d.o.f. of the tool.

Without a lack of generality, the 1D case is presented here for clarity reasons. Following results can be immediately extended to $2 \mathrm{D}$ or $3 \mathrm{D}$. Lets suppose one node in contact with a force driven tool. The tangent stiffness matrix of this force element is written

$$
K_{\mathrm{T}}=\left(\begin{array}{cc}
K_{b b} & K_{b M} \\
K_{M b} & K_{M M}
\end{array}\right)=\left(\begin{array}{lll}
\frac{\partial F_{b_{i}}^{\text {int }}}{\partial x_{b_{i}}}-\frac{\partial F_{b_{i}}^{\text {ext }}}{\partial x_{b_{i}}} & \frac{\partial F_{b_{i}}^{\text {int }}}{\partial x_{M}}-\frac{\partial F_{b_{i}}^{\text {ext }}}{\partial x_{M}} \\
\frac{\partial F_{M}^{\text {int }}}{\partial x_{b_{i}}}-\frac{\partial F_{M}^{\text {ext }}}{\partial x_{b_{i}}} & \frac{\partial F_{M}^{\text {int }}}{\partial x_{M}}-\frac{\partial F_{M}^{\text {ext }}}{\partial x_{M}},
\end{array}\right)
$$

where $x$ is the coordinate of a d.o.f., the subscripts $M$ refer to the tool and the subscripts $b$ refer to the node in contact with the blank-holder.

If the desired force is denoted $F$, the internal and external forces of the tool can be rewritten:

$$
\begin{aligned}
& F_{M}^{\mathrm{int}}=-F \\
& F_{M}^{\mathrm{ext}}=-\sum_{k=1}^{n c_{b}} F_{b_{k}}^{\mathrm{ext}} .
\end{aligned}
$$

Consequently, the tangent stiffness matrix can be entirely rewritten using the quantity $R T C=-\frac{\partial F_{b_{k}}^{\text {ext }}}{\partial x_{b_{k}}}$. The latter was already computed by METAFOR for the stiffness matrix of the contact element by the penalty method. Finally, a classical stiffness matrix is obtained

$$
K_{\mathrm{T}}^{b_{i}}=\left(\begin{array}{cc}
R T C & -R T C \\
-R T C & R T C
\end{array}\right) .
$$

This elementary matrix is assembled into the system stiffness matrix and the residual force associated to the new d.o.f. is assembled into the right-hand side of the linearized system to be solved.

Once convergence is obtained, the equilibrium equations of the deformable body are satisfied as well as the equilibrium of the force driven tool.

\section{Contact algorithms}

We roughly describe, in this section, the different steps of our algorithms detailed in the previous sections. They all follow the same main algorithm (Fig. 1) used to solve incremental nonlinear problems. 


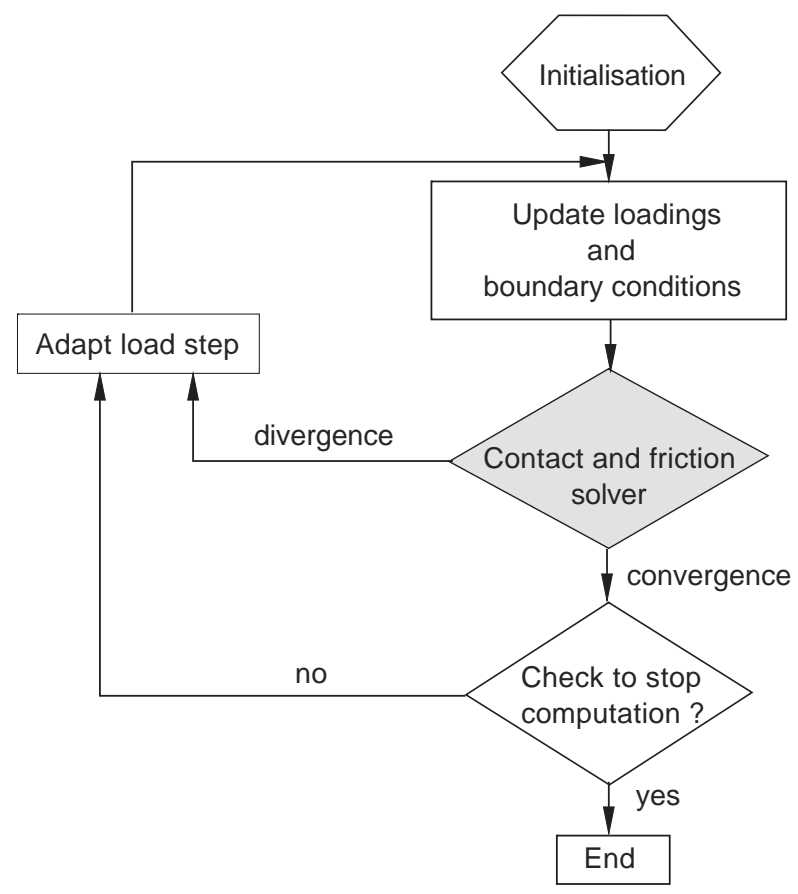

Fig. 1. Main algorithm.

\subsection{LMA contact and friction solver}

On the flowchart represented in Fig. 2 we have summarized the main aspects of our contact solver. One can observe that non-linearities coming from contact are uncoupled of those coming from volumic behavior (i.e., large strains, non-linear material behavior).

This is a "local" solver, which means that all the non-linearities coming from contact and friction are treated in the Lemke's solver itself.

\subsection{LTAS contact and friction solver}

On the two flowcharts represented in Figs. 3 and 4 we have summarized the main aspects of the two solvers used by the LTAS for the present work. One can obviously consider the penalty solver as a particular case of the Augmented Lagrangian one.

These two approaches are "global" solvers consisting in introducing the contact non-linearities at the upper level (i.e., the stiffness matrix) and using a classical linear system solver.

\subsubsection{Penalty algorithm}

Penalization algorithm is shown in the flowchart (Fig. 3).

\subsubsection{Augmented Lagrangian algorithm}

Augmented Lagrangian algorithm is shown in the flowchart (Fig. 4). 


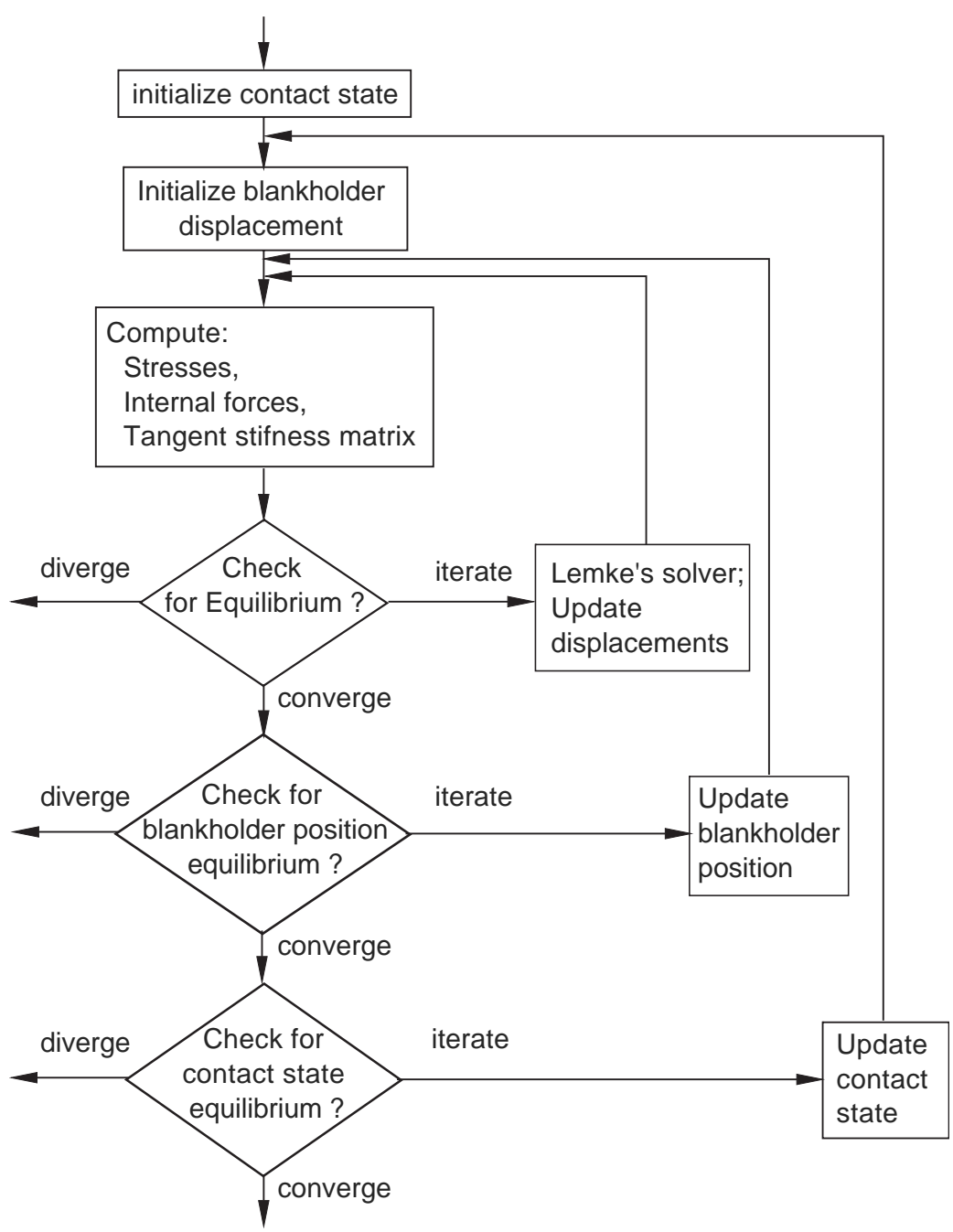

Fig. 2. Lemke's solver.

\section{Numerical simulation of tribological devices}

\subsection{Introduction}

We present below the results of some simulations that have been carried out in parallel by the two softwares. All considered materials are elasto-plastic with isotropic hardening. Locally, the classical Coulomb friction law with constant friction coefficient has been used so that, in essence, the main difference is the geometry of tools that have been compared. All these tools are considered to be rigid and the analytical description of all their surfaces has been used throughout all the computations. 


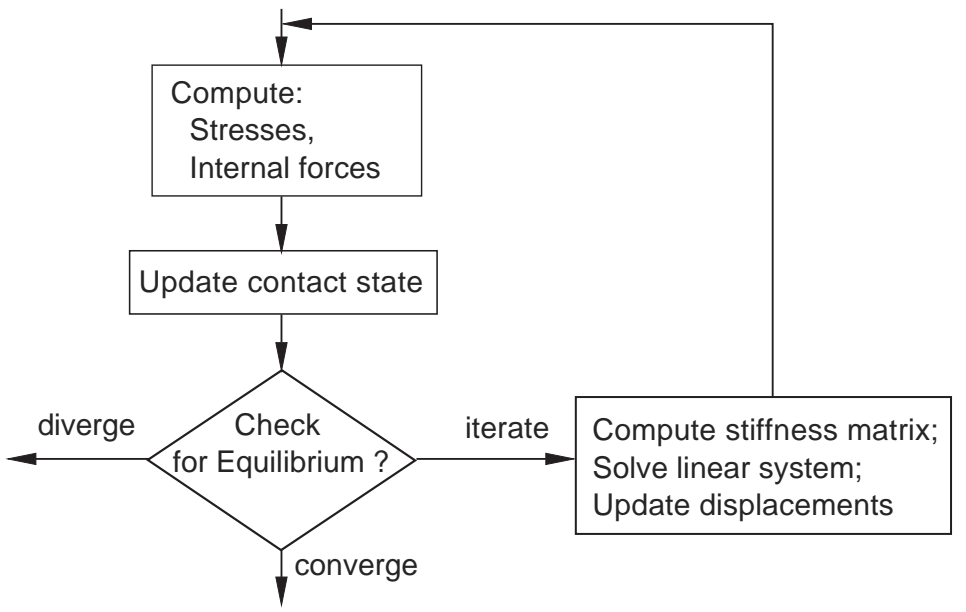

Fig. 3. Penalization algorithm.

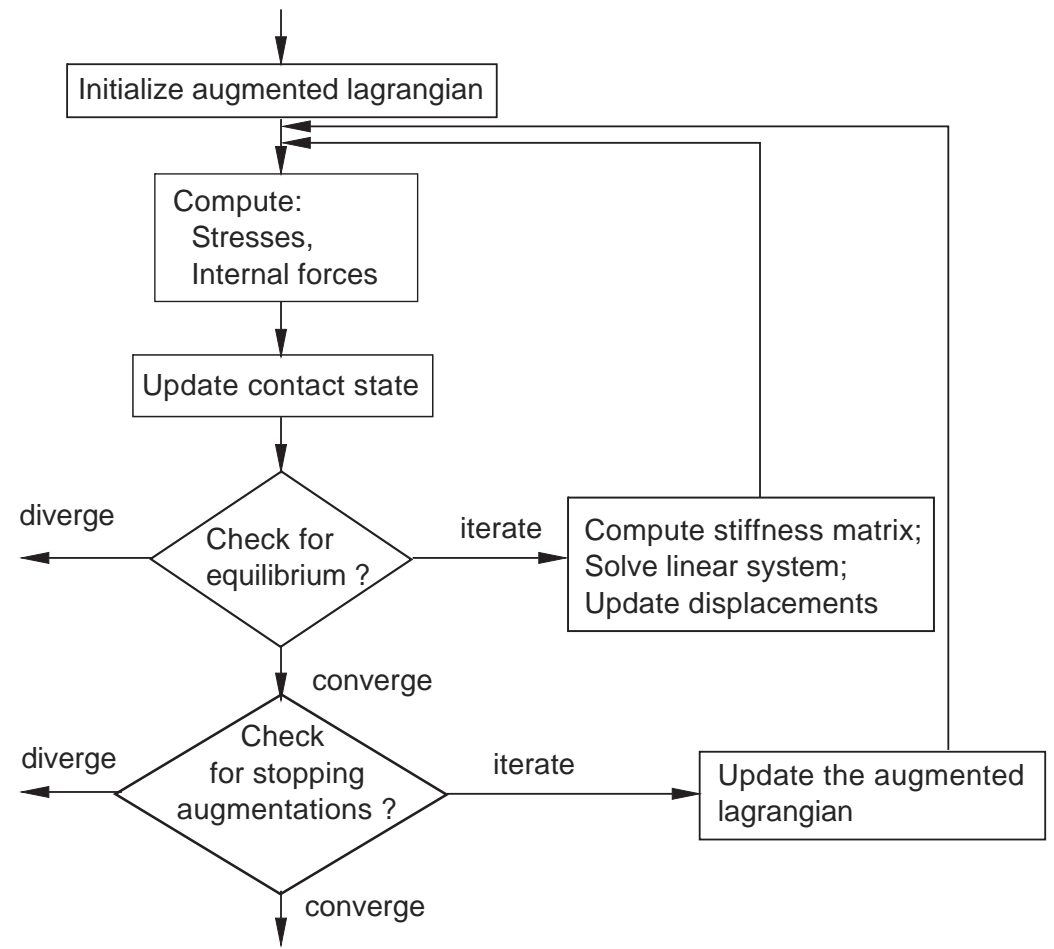

Fig. 4. Augmented Lagrangian algorithm.

In all examples the clamping of the sheet has been applied through the tooling. This one has been submitted to prescribed tool displacements or constant clamping forces applied through the previously described algorithms. Emphasize is put on the state of contact, i.e., the distribution of contact forces along 


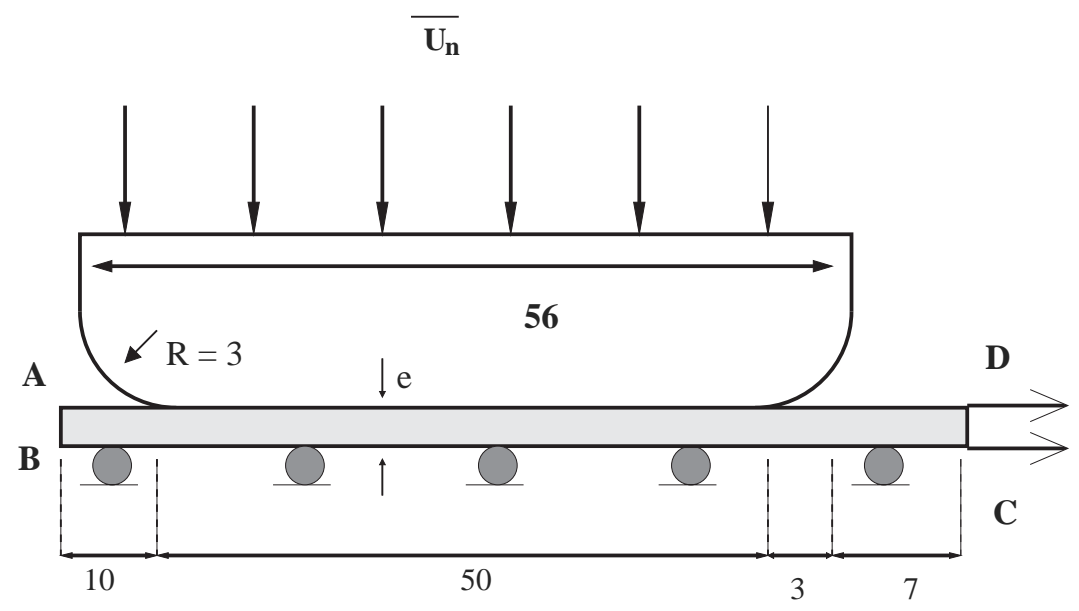

Fig. 5. Flat die test geometry. Problem description.

Table 1

Material properties for the flat die test

\begin{tabular}{ll}
\hline Young's Modulus & $E=69004$ \\
Poisson's ratio & $v=0.3$ \\
Hardening behavior & $\sigma_{\mathrm{v}}=80.55923(1+10000 \overline{\bar{\varepsilon}} \mathrm{p})^{0.216}$ \\
\hline
\end{tabular}

contact zones. Special attention has also been carried when particular methodologies lead to different numerical results.

\subsection{Flat die test}

This first example seems very simple. It consists of a rigid flat die, whose geometry is given in Fig. 5. First the tool is moved down to characterize the clamping. When a clamping force of $50 \mathrm{~N}$ per millimeter out of the plane is reached, the second stage, consisting of the drawing, is accounted for by a prescribed horizontal incremental displacement on the right-hand side of the sheet (edge DC) until a steady state of sliding is obtained.

Two drawing cases have been considered. In the first one the constant vertical clamping force is maintained constant during the drawing phase thanks to the algorithms described in Sections 5.1 and 5.2. In the second case, the vertical position reached by the tool at the end of the clamping phase is maintained constant in position during the drawing phase, allowing the vertical force to vary.

The test-piece is a $0.79 \mathrm{~mm}$ thick sheet. Because of the symmetry, the actual thickness analyzed corresponds to a half width $(0.395 \mathrm{~mm})$ and the vertical displacements are imposed to be zero on $\mathrm{BC}$. The mesh consists of 480 finite elements (2 layers of 240 elements) which are initially identical. The material is supposed to behave as an elasto-plastic material with nonlinear isotropic hardening as described in Table 1. 


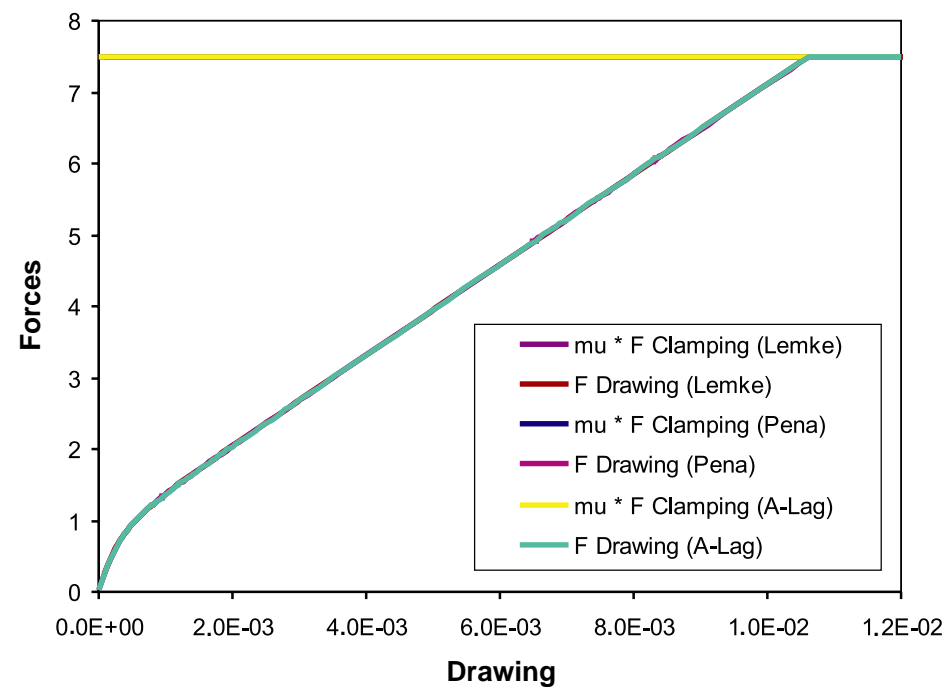

Fig. 6. Flat die test, constant clamping force, global contact forces.

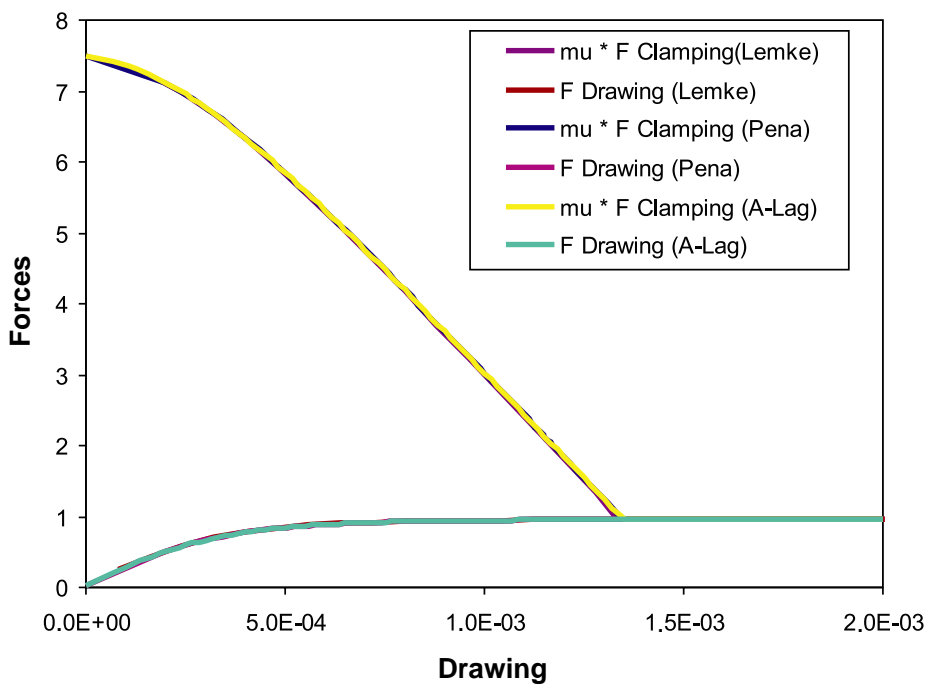

Fig. 7. Flat die test, constant tool displacement, global contact forces.

The unilateral conditions and the friction law, corresponding to a Coulomb model with $\mu=0.15$ is considered on AD. Penalty coefficients are respectively set to $\varepsilon_{n}=10^{9} \mathrm{~N} / \mathrm{mm}$ and $\varepsilon_{t}=0.15 \times 10^{9} \mathrm{~N} / \mathrm{mm}$.

Figs. 6 and 7 show the global contact state for respectively constant clamping force and constant tool position. These global forces are obtained by the summation on all nodal contact forces.

The curves have been plotted for all three contact algorithms. The results are almost identical. In Fig. 6, it can be observed that the normal clamping force is constant, and equal to the prescribed value, 

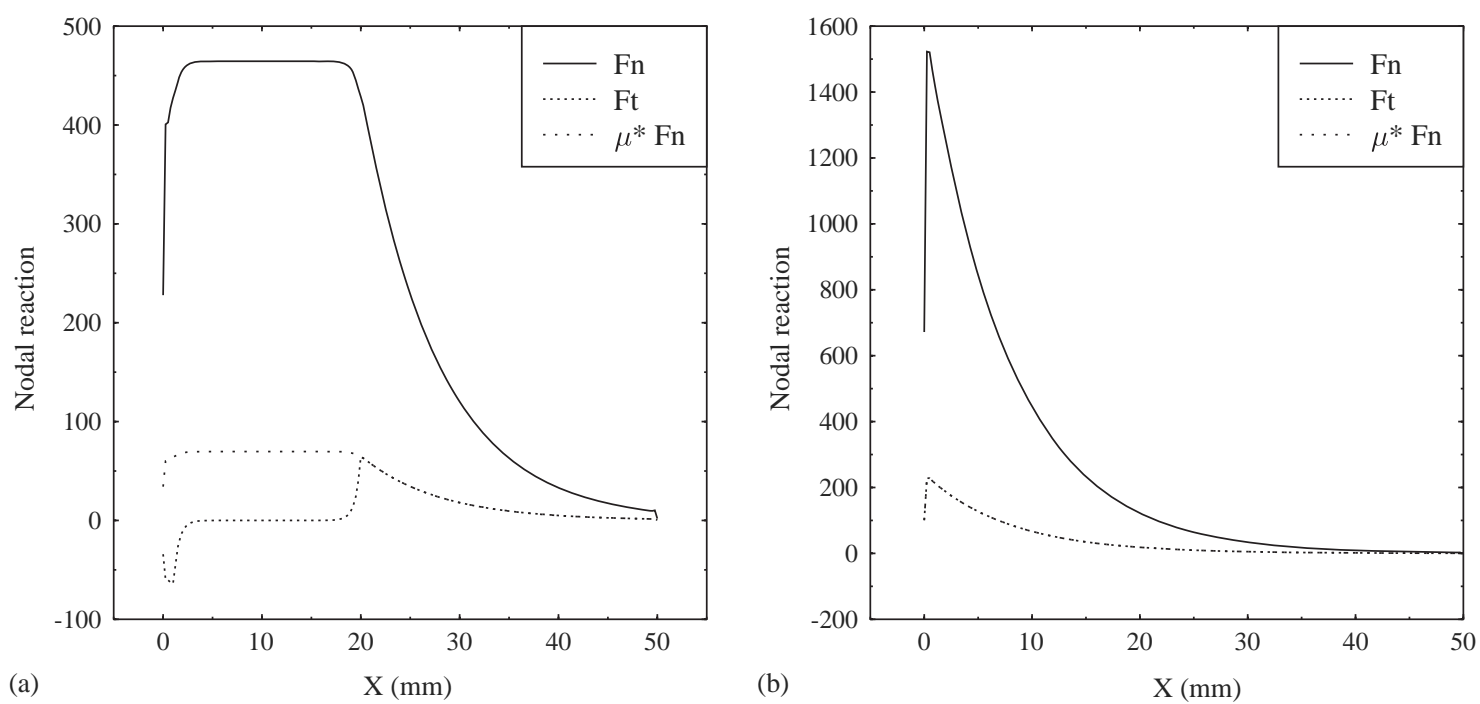

Fig. 8. Flat die test, constant clamping force. Contact forces distribution: (a) intermediate configuration, (b) global sliding.
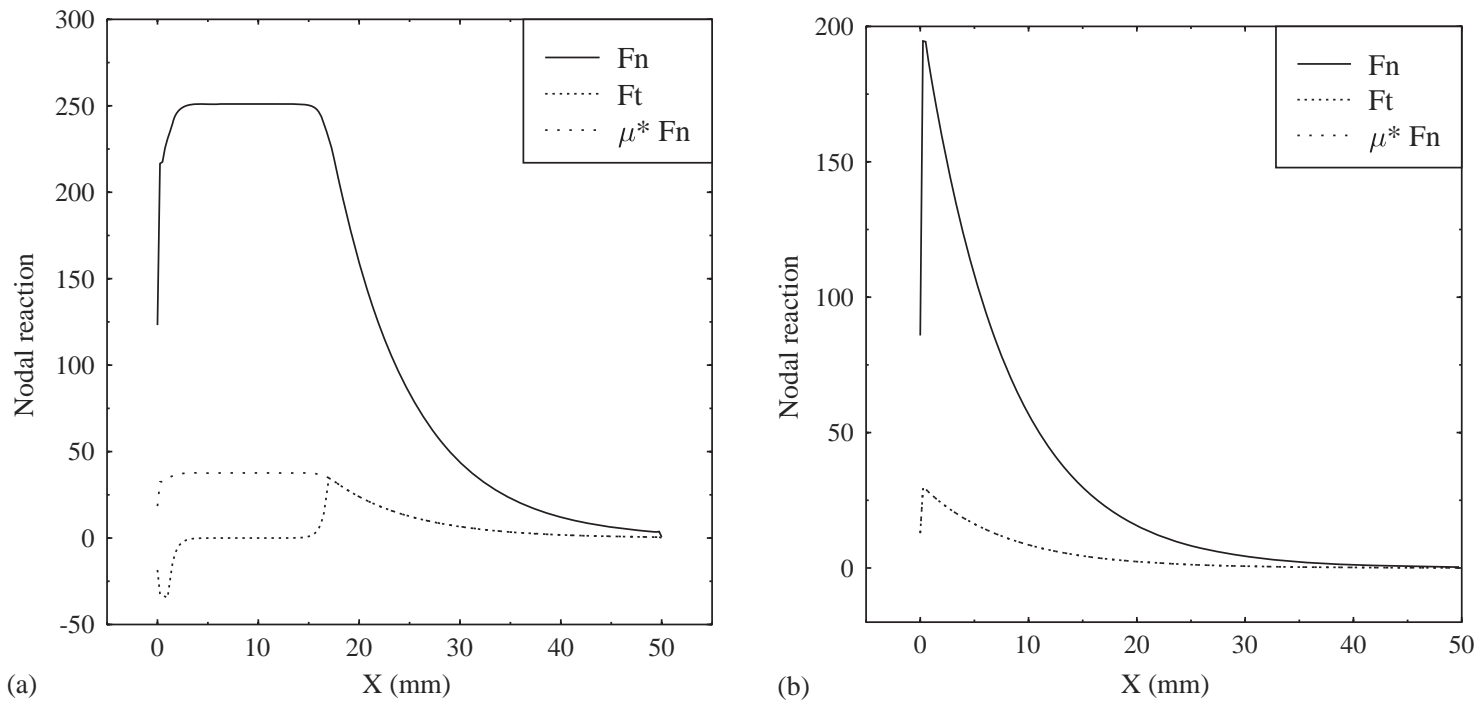

Fig. 9. Flat die test, constant tool displacement. Contact forces distribution: (a) intermediate configuration, (b) global sliding.

throughout the simulation. Global sliding occurs after a drawing displacement of $0.01064 \mathrm{~mm}$. For the case of constant tool displacement (Fig. 7), the normal force decreases from 50-6.37 N very rapidly and then remains constant. When it reaches the minimum value, the drawing displacement is equal to $0.00136 \mathrm{~mm}$ and, at this stage, global sliding occurs.

Figs. 8 and 9 give, at a given stage of the drawing, for both loading cases, the distribution of the nodal contact forces along the contact area for two stages in the drawing process. On these figures, a third curve, 


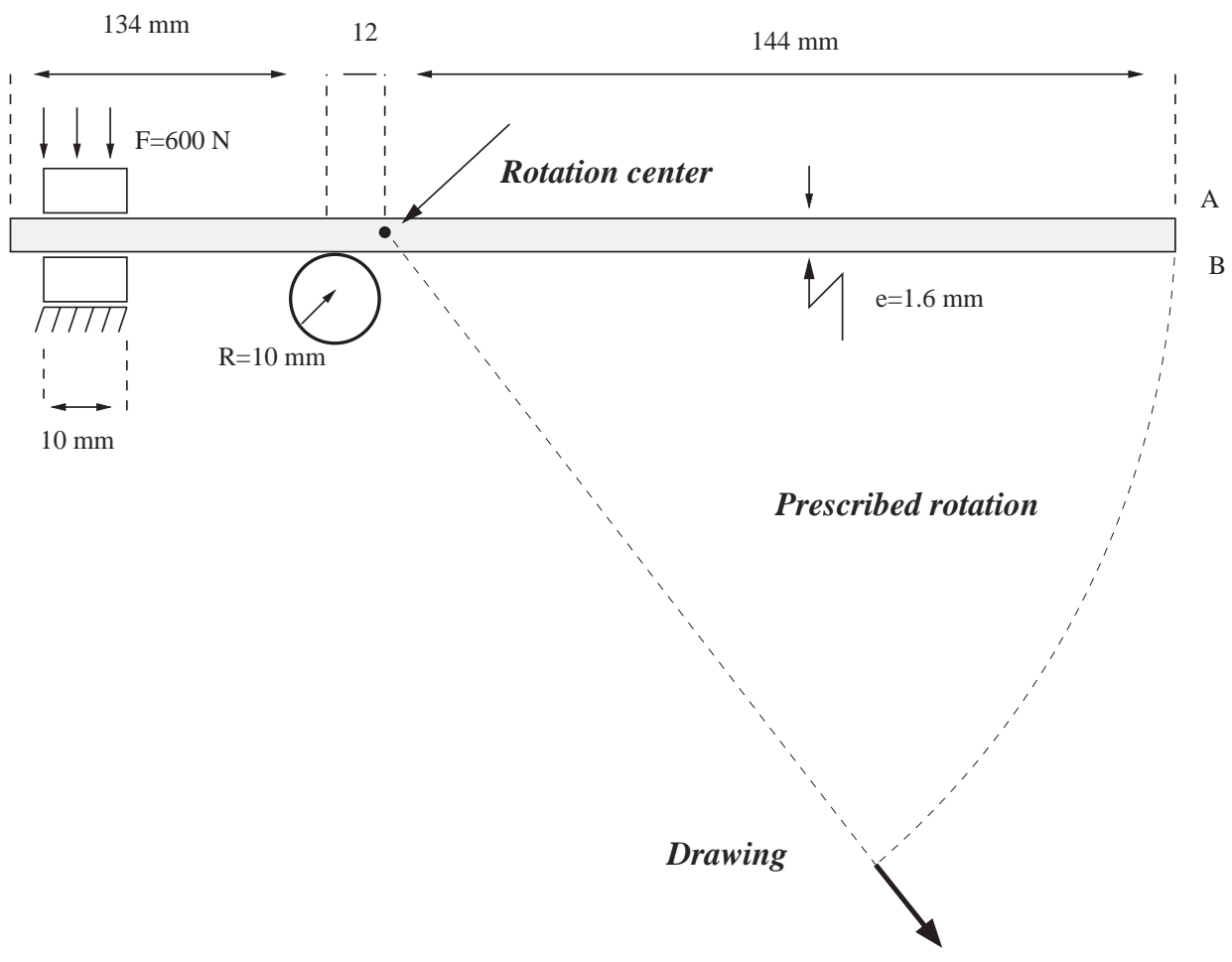

Fig. 10. Bead test description. Initial geometry.

which is the product of the friction coefficient times the global normal force has also been plotted. When this curve is identical to the tangential one, sliding occurs.

Due to a very small decrease in the sheet thickness on its right-hand side, the area of the sheet held by the clamp decreases (see Figs. 8 and 9) with the drawing process. The global sliding of the whole piece occurs very soon. It is a stable state which corresponds to a global Coulomb friction behavior. When this global sliding occurs, the normal force is carried by only a small part of the nominal area of contact.

\subsection{Friction on bead test}

This test principle which is given in Fig. 10, consists of: two flat dies (left) which apply a constant clamping force, a central roller around which the test piece is wound, and two jaws (right, not represented) which impose the winding and drawing on right edge.

In the first phase, the test-piece is clamped on its left hand side by the flat dies until a clamping force of $600 \mathrm{~N}$ per millimeter out of the plane is reached. This clamping force is then maintained constant during the remaining phases. The friction coefficient between the sheet and the clampers is assumed to be $\mu=0.17$ all along the simulations.

In a second phase, the jaws prescribe on the test-piece a rotation around the central roller. When the required rolling-up angle has been reached, the third phase starts. The test-piece is drawn along $8 \mathrm{~mm}$ 
Table 2

Material properties for the bead test

\begin{tabular}{ll}
\hline Young's Modulus & $E=200000$ \\
Poisson's ratio & $v=0.3$ \\
Hardening behavior & $\sigma_{\mathrm{v}}=169\left(1+127.42425 \bar{\varepsilon}^{\mathrm{p}}\right)^{0.225}$ \\
\hline
\end{tabular}
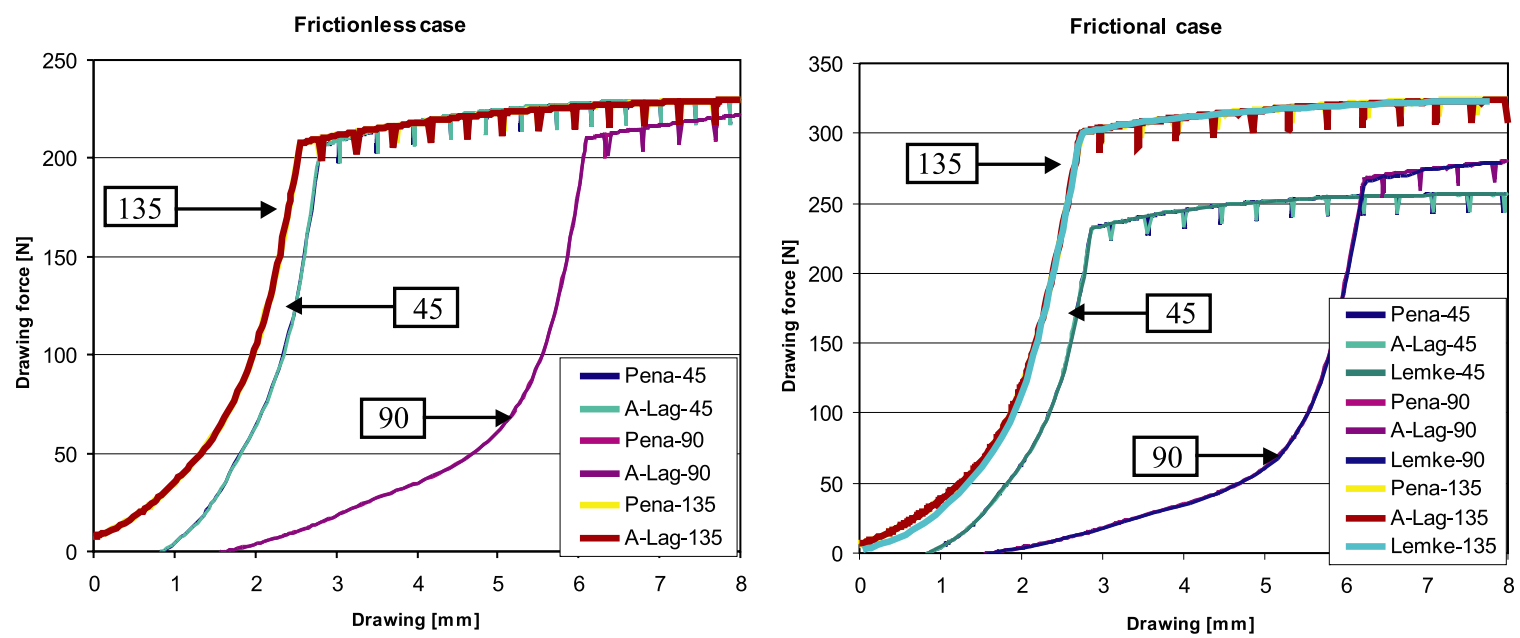

Fig. 11. Bead test: drawing forces.

without any further angle variation. With this device it is possible to change the restraining forces (by adjusting the clamping pressure and the friction between the flat dies), the roller radius, the rollingup angle and the pulling speed. In these simulations, two friction coefficients between the sheet and the central roller have been considered, respectively $\mu=0$ and 0.17 . Three rotation angles around the central roller have also been considered: $\theta=45^{\circ}, 90^{\circ}$ and $135^{\circ}$. The mesh consists of 4 layers of 725 initially identical elements. The material is supposed to behave as an elasto-plastic material with nonlinear isotropic hardening as described in Table 2.

Fig. 11 shows the global restraining forces. Here the contact state is strongly correlated with the specific characteristics of the problem. As a matter of fact, non-constant and constant pressure lead to conspicuously different results. In the first case, the one with nonconstant pressure, the contact on the roller is very weak at the beginning of the drawing process. The distribution of the contact forces obviously depends on the geometry involved specially on the roller radius, the sheet length and the sheet thickness (Figs. 12-15).

\subsection{Radial strip drawing test}

The corresponding experimental device was first proposed by Nine [7-9]. Here the aim was to design an experiment in which it would be possible to distinguish within a process the forces resulting from bending/unbending of the sheet from forces due to friction. The geometry of this test is described in 


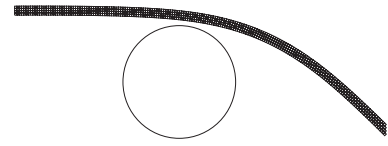

(a)

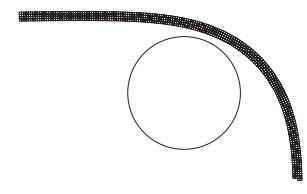

(b)

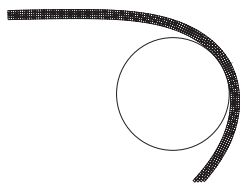

(c)

Fig. 12. Bead test with friction. Contact state after folding: (a) $\theta=45^{\circ}$, (b) $\theta=90^{\circ}$, (c) $\theta=135^{\circ}$.

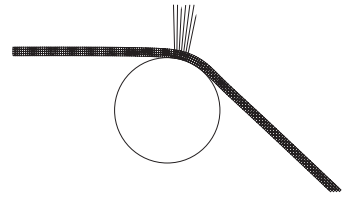

(a)

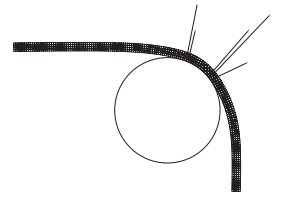

(b)

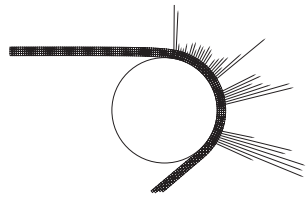

(c)

Fig. 13. Bead test with friction. Contact state during drawing: (a) $\theta=45^{\circ}$, (b) $\theta=90^{\circ}$, (c) $\theta=135^{\circ}$.

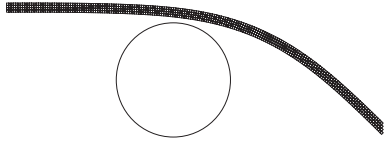

(a)

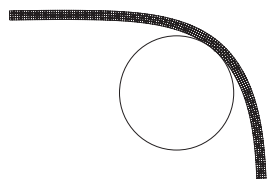

(b)

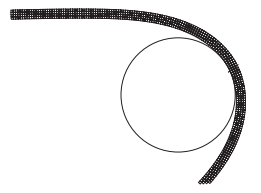

(c)

Fig. 14. Bead test frictionless. Contact state after folding: (a) $\theta=45^{\circ}$, (b) $\theta=90^{\circ}$, (c) $\theta=135^{\circ}$.

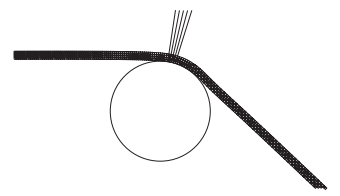

(a)

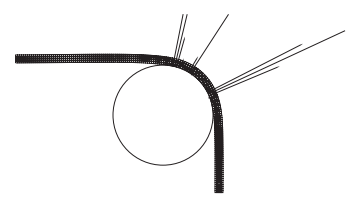

(b)

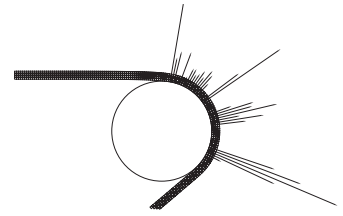

(c)

Fig. 15. Bead test frictionless. Contact state during drawing: (a) $\theta=45^{\circ}$, (b) $\theta=90^{\circ}$, (c) $\theta=135^{\circ}$.

Fig. 16 and the corresponding dimensions are given in Table 3. The process is divided into a clamping phase, in which the upper bead, called the clamping bead, is moved downwards $11 \mathrm{~mm}$ (corresponding to the cylinder diameter $2 R_{1}$ ) between the groove shoulders, and a drawing phase in which the bead remains motionless (no translation) while the sheet is drawn. In the frictionless case, the cylinders are allowed to roll in order to eliminate the friction effects whereas in the frictional case they remain fixed. 


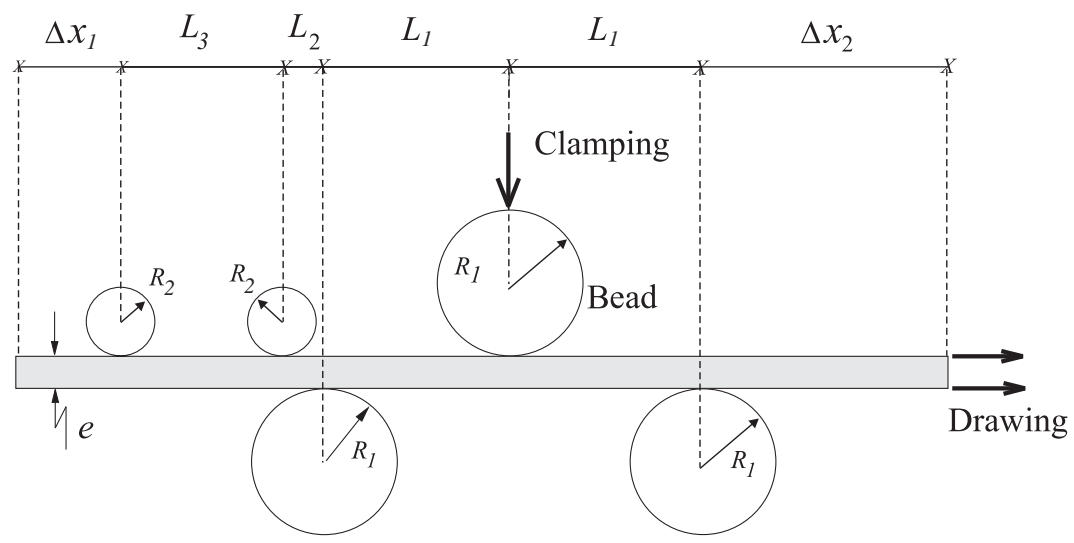

Fig. 16. Radial strip drawing test geometry - initial configuration.

Table 3

Dimensions for the radial strip drawing $(\mathrm{mm})$

\begin{tabular}{|c|c|c|c|c|c|c|c|}
\hline$R_{1}$ & $R_{2}$ & $L_{1}$ & $L_{2}$ & $L_{3}$ & $e$ & $\Delta x_{1}$ & $\Delta x_{2}$ \\
\hline 5.5 & 2.87 & $2 R_{1}+e$ & 2.63 & 12.43 & 0.97 & 50 & $100-\Delta x_{1}-2 L_{1}-L_{2}-L_{3}$ \\
\hline
\end{tabular}

Table 4

Material properties for the radial strip drawing

\begin{tabular}{ll}
\hline Young's Modulus & $E=200000$ \\
Poisson's ratio & $v=0.3$ \\
Hardening behavior & $\sigma_{\mathrm{V}}=171(1+121.74485 \bar{\varepsilon} \mathrm{p})^{0.23}$ \\
\hline
\end{tabular}

Further, the metal sheet under investigation is drawn for a sufficiently long time to reach a steady state as far as the drawing force is concerned.

The two small cylinders (radius $R_{2}$ ) are allowed to rotate freely and consequently, the contact can be assumed frictionless on these two rollers. The contact between the sheet and the three other cylinders is assumed to be frictionless (cylinders free to rotate) or to follow the Coulomb model with a friction coefficient of $\mu=0.17$. The material is supposed to behave as an elasto-plastic material with nonlinear isotropic hardening as described in Table 4. Unless otherwise stated, the sheet is meshed using $n_{x}=300$ elements along the drawing direction and $n_{y}=3$ elements through the thickness.

In Figs. 17 and 18 the restraining forces have been plotted for both frictionless and frictional cases. The clamping force is the vertical reaction on the clamping bead while the drawing force is the horizontal reaction at the right edge of the sheet. In Fig. 18, one can see that, after some transient evolution, a steady state is reached. After that (in the neighborhood of $60 \mathrm{~mm}$ drawing), the forces drop suddenly. This corresponds to the left side of the sheet being swallowed in the bead system. 

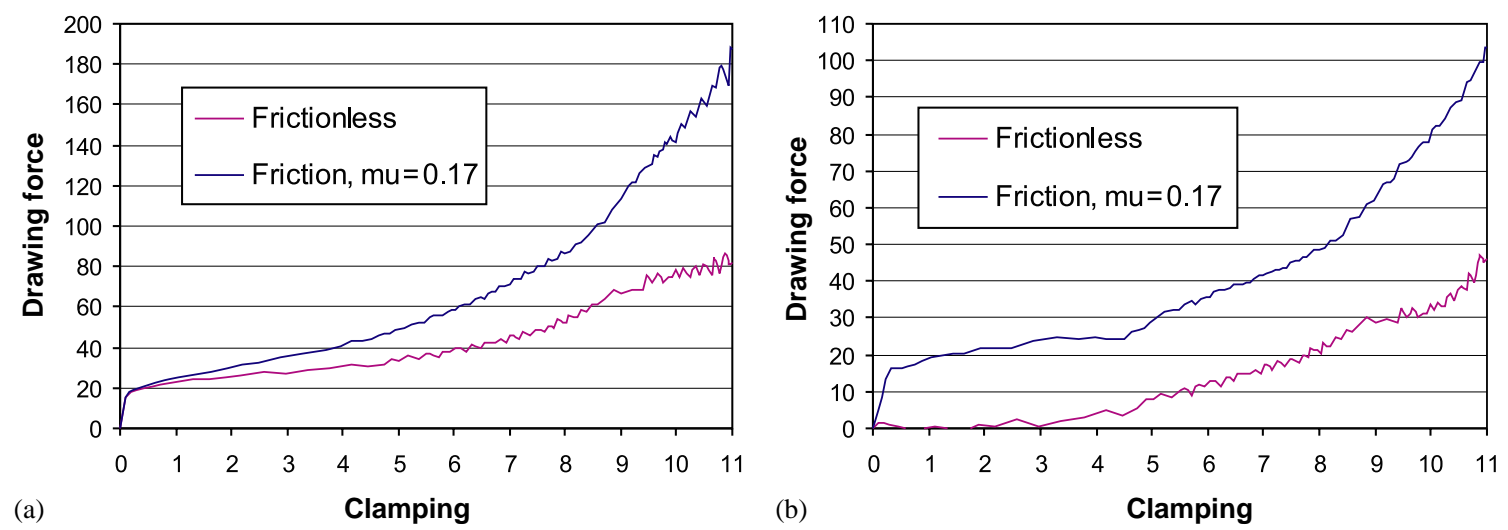

Fig. 17. Radial strip drawing test: clamping phase: (a) $F_{X}$, (b) $F_{Y}$.
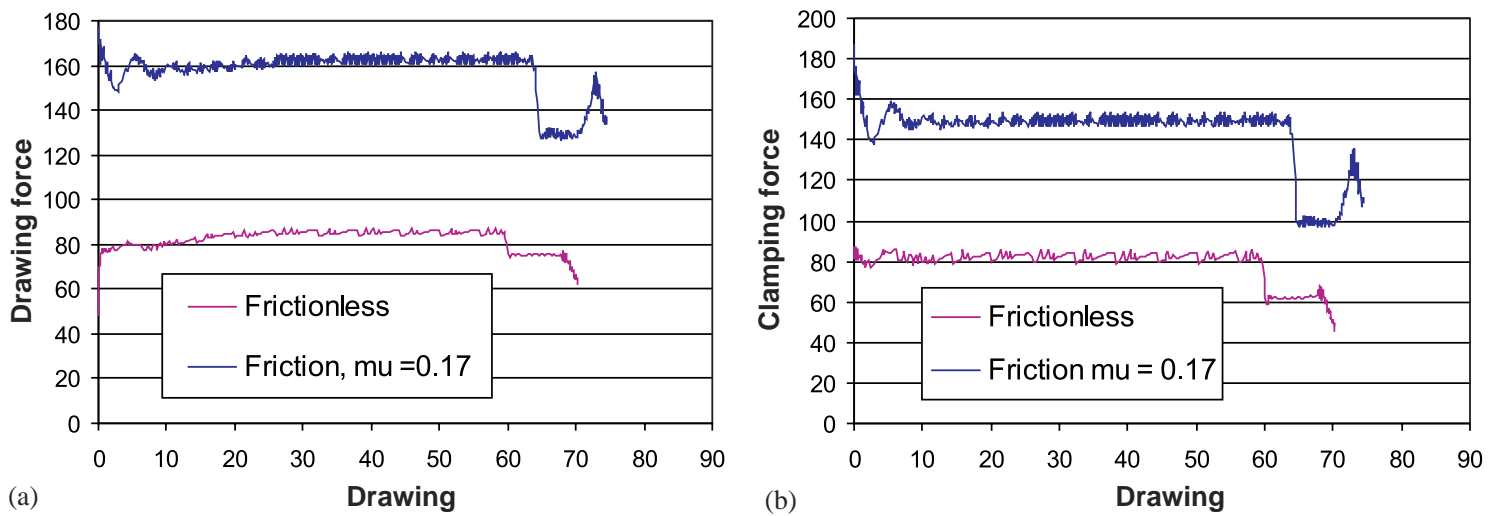

Fig. 18. Radial strip drawing test: drawing phase: (a) $F_{X}$, (b) $F_{Y}$.

In Fig. 19, the reaction forces given by the three contact algorithms have been plotted (frictional case). Once again, the results are quite similar. The distribution and the intensity of the contact forces are given in Fig. 20, where each arrow corresponds to a contact reaction. The length of an arrow is proportional to the intensity of the local contact force.

Fig. 21 shows the mesh size dependence of the solution. The penalty method is used in the frictionless case. The penalty coefficient is $10^{5} \mathrm{~N} / \mathrm{mm}$. The mean drawing force is plotted for several discretizations along the length of the sheet (150, 300, 600 and 1200 elements) and two discretizations $n_{y}=3$ and $n_{Y}=6$ elements through the thickness. This shows that our reference mesh $(300 \times 3$ elements) already gives a fairly good solution (4\% of error compared to the finer mesh) for a reasonable CPU cost. This graph also shows that, from an engineering point of view, 3 elements through the thickness are a good trade to model accurately the bending of the sheet. As far as the computation time is concerned, our tests show the increase of the CPU time with the mesh size-see Fig. 21. Once again, the reference mesh seems to be a good compromise between accuracy and speed. 

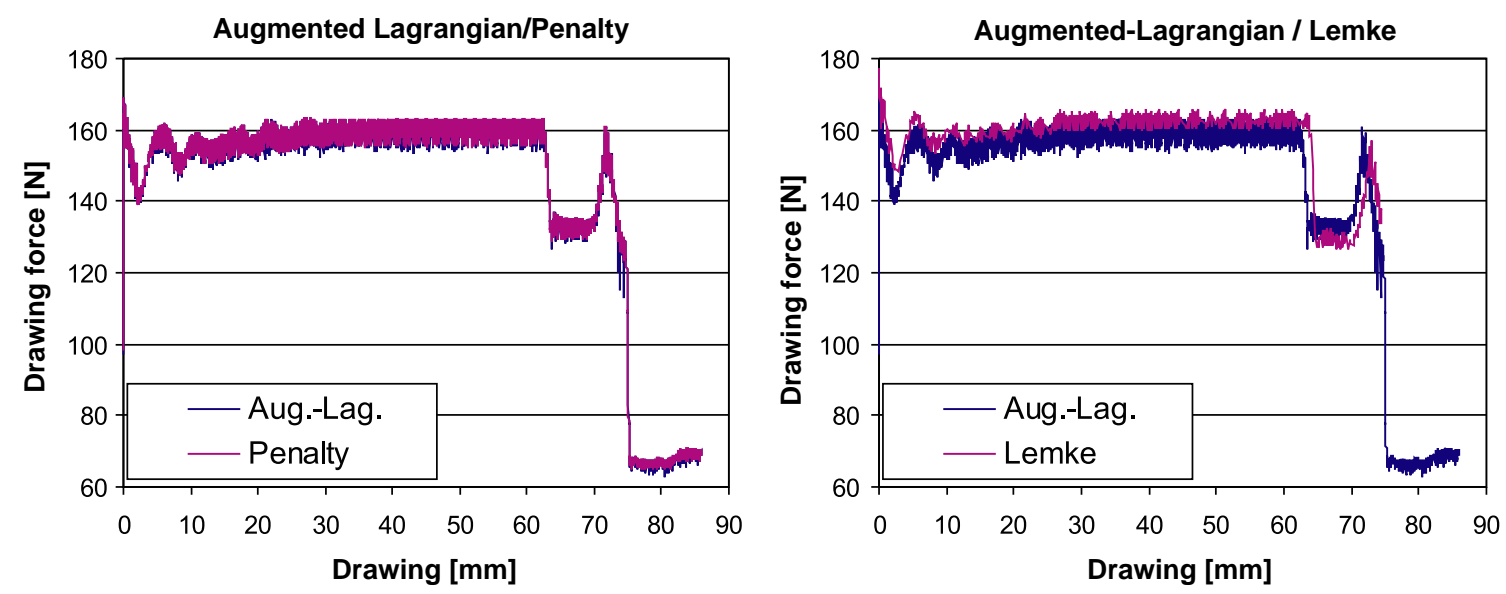

Fig. 19. Comparison of drawing forces for different contact algorithms (frictional case).
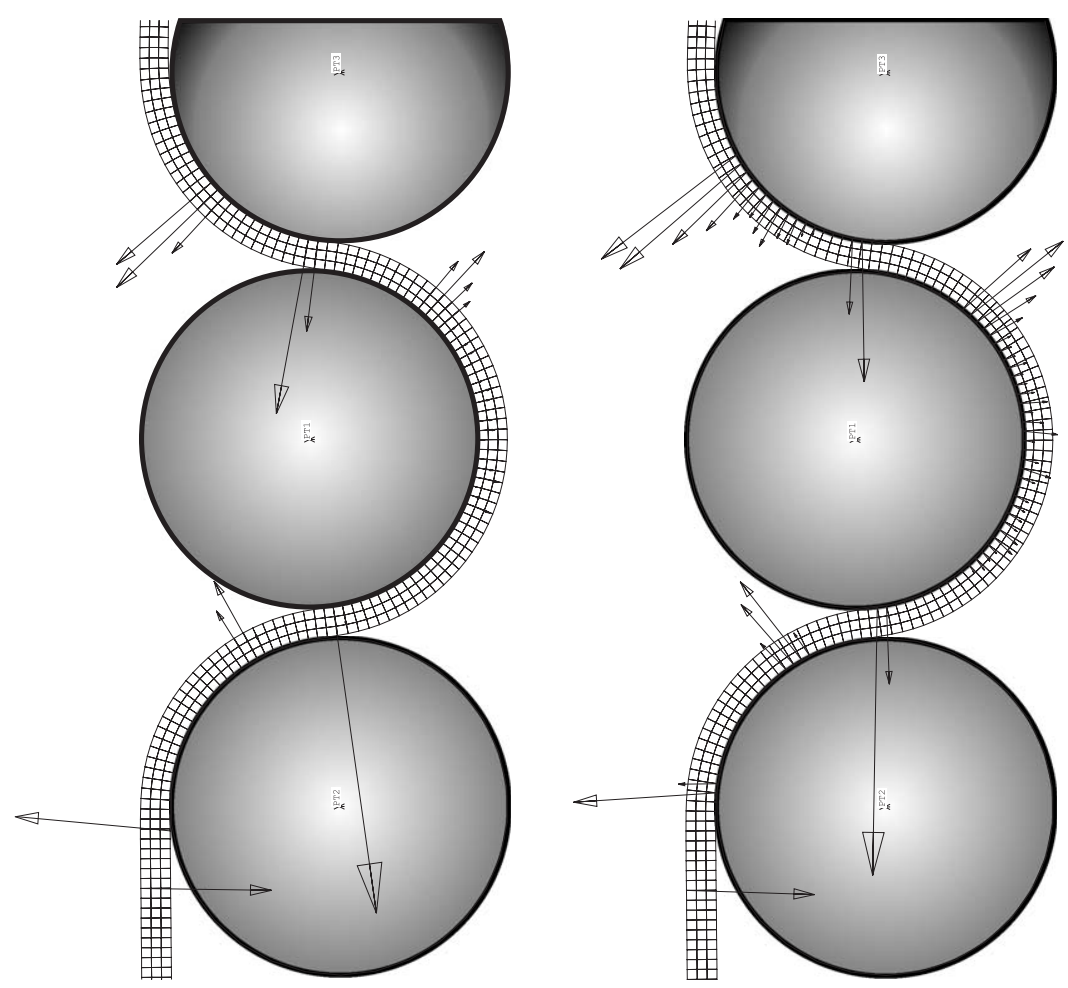

Fig. 20. Radial strip drawing test, frictionless (left) and frictional (right) contact states during stabilized phase.

One other interesting point is the sensitivity of the solution accuracy with respect to the penalty parameters and the required precision. Tables 5 and 6 give an idea of the evolution of the solution cost and accuracy versus the normal penalty parameter as well as the required accuracy (target gap in the 

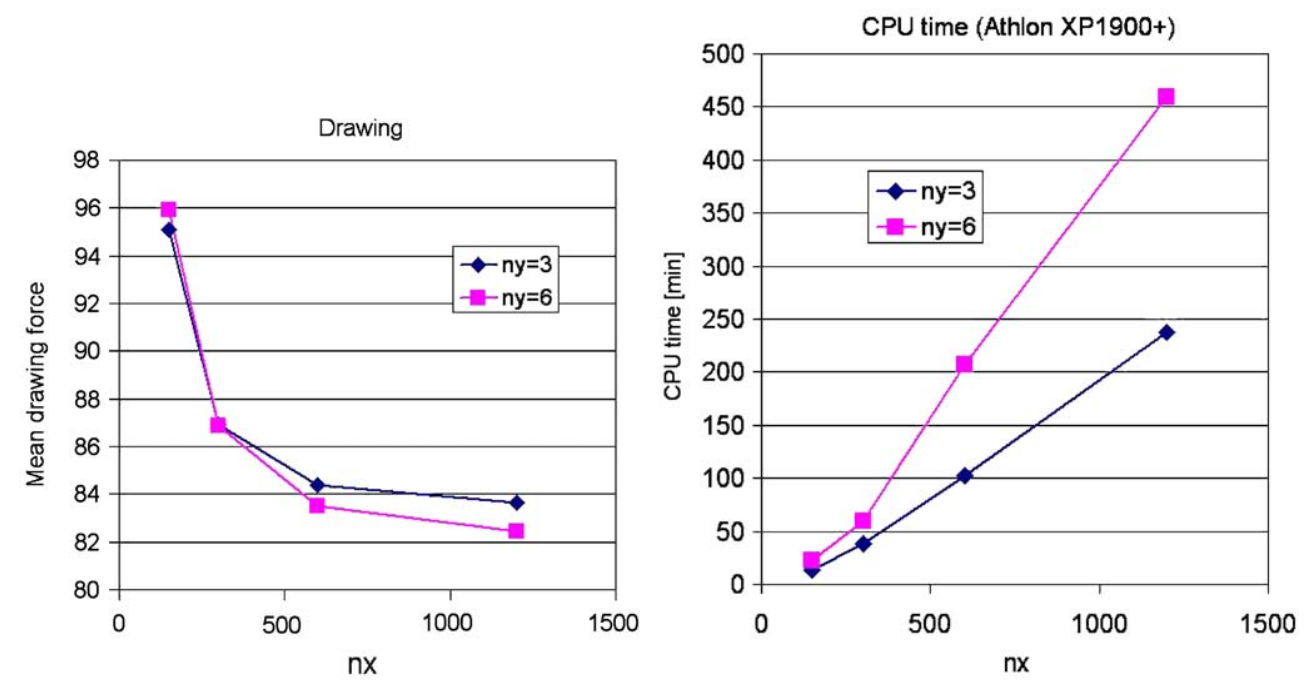

Fig. 21. Sensitivity of the solution to the mesh-frictionless case. Penalty algorithm.

Table 5

Solution sensitivity to Penalty parameter

\begin{tabular}{|c|c|c|c|c|}
\hline$\varepsilon_{n}$ & Time step & Iterations & $\mathrm{CPU}$ & MaxGap \\
\hline \multicolumn{5}{|c|}{ Frictionless } \\
\hline 1.E+02 & 1919 & 6331 & 1.00 & 2.E-01 \\
\hline 1.E+03 & 2434 & 7524 & 1.15 & 6.E-02 \\
\hline 1.E+04 & 3255 & 10181 & 1.51 & 9.E-03 \\
\hline 1.E+05 & 8045 & 24343 & 4.19 & 1.E-03 \\
\hline 1.E+06 & 25544 & 74413 & 10.71 & 1.E-04 \\
\hline \multicolumn{5}{|c|}{ Friction $\mu=0.17$} \\
\hline 1.E+02 & 2005 & 6464 & 1.01 & 2.E-01 \\
\hline 1.E+03 & 2512 & 7692 & 1.16 & 6.E-02 \\
\hline 1.E+04 & 3509 & 11008 & 1.60 & 8.E-03 \\
\hline 1. $E+05$ & 8425 & 26799 & 3.83 & 1.E-03 \\
\hline 1.E+06 & 26761 & 82075 & 11.76 & 1.E-04 \\
\hline
\end{tabular}

case of the Augmented Lagrangian algorithm). In these tables, the CPU time has been normed with respect to the CPU time for the frictionless case with $\varepsilon_{n}=1 . E+02$. In the case of frictional contact, the tangent penalty parameter is always taken as the friction coefficient times the friction coefficient, i.e., $\varepsilon_{t}=\mu \varepsilon_{n}$.

For the Penalty case, it can be noted that, as the penalty coefficient is raised, the CPU time increases and the maximum gap decreases, as can be expected. However, it can also be noted that a 
Table 6

Solution sensitivity for the Augmented Lagrangian

\begin{tabular}{|c|c|c|c|c|c|}
\hline$\varepsilon_{n}$ & Target gap & Time steps & Iterations & $\mathrm{CPU}$ & Aug. \\
\hline \multicolumn{6}{|c|}{ Frictionless } \\
\hline 1.E+04 & 1.E-04 & 3444 & 40480 & 6.09 & 3.84 \\
\hline 1. $E+05$ & $1 . E-05$ & 7749 & 43641 & 6.81 & 1.37 \\
\hline 1.E+05 & 1.E-06 & 7918 & 60538 & 9.98 & 3.37 \\
\hline \multicolumn{6}{|c|}{ Friction $\mu=0.17$} \\
\hline 1.E+04 & 1.E-03 & 3157 & 17153 & 2.66 & 0.61 \\
\hline 1.E+04 & 1.E-04 & 3645 & 40319 & 5.83 & 3.51 \\
\hline 1.E+05 & $1 . \mathrm{E}-06$ & 8400 & 72307 & 10.94 & 3.88 \\
\hline
\end{tabular}
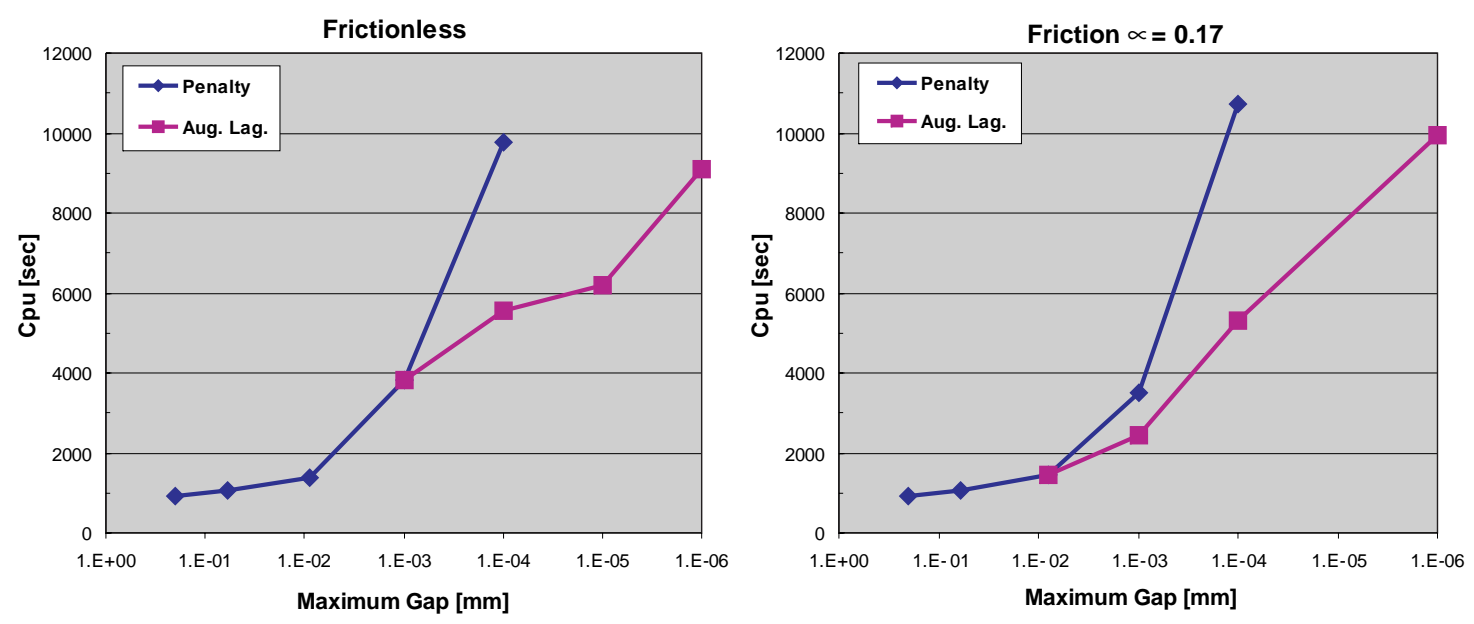

Fig. 22. CPU versus maximum gap for the Nine problem.

large variety of penalty coefficients can be used without affecting too much the drawing of the curves since for $\varepsilon_{n} \geqslant 1 . E+03$, the maximum gap all along the deformation is smaller than $1 \%$ of the bead radius.

In the Augmented Lagrangian case, the augmentations are performed automatically until the maximum gap is smaller than the user defined Target Gap. In Table 6 the column Aug. gives the average number of augmentations during a time step.

The evolution of the CPU cost, as a function of the maximum gap is also plotted in Fig. 22. On these figures, it is interesting to note that the Augmented Lagrangian curve is always lower than the Penalty 


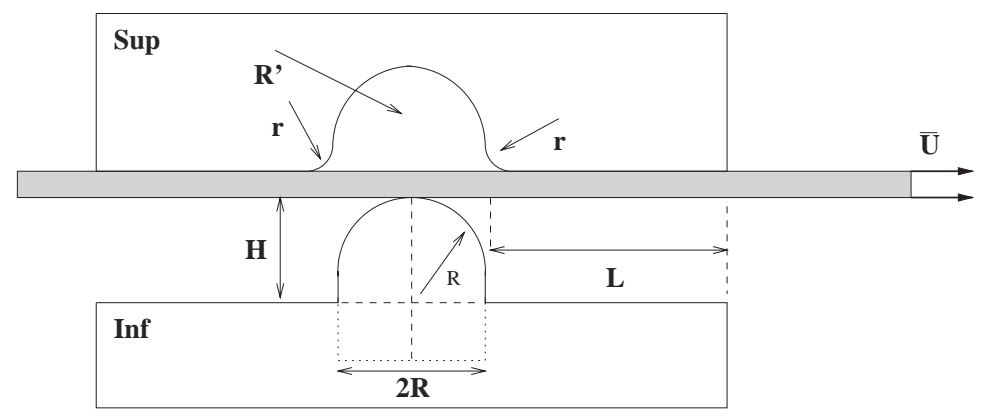

Fig. 23. Blankholder with drawbead test geometry.

Table 7

Table of the various geometries

\begin{tabular}{llllll}
\hline Shape & $H(\mathrm{~mm})$ & $R(\mathrm{~mm})$ & $r(\mathrm{~mm})$ & $R^{\prime}(\mathrm{mm})$ & $L(\mathrm{~mm})$ \\
\hline 1 & 6 & 6 & 3 & 7.25 & 25 \\
2 & 6 & 6 & 3 & 10.2 & 50 \\
3 & 9 & 6 & 4.5 & 10.2 & 25 \\
4 & 9 & 6 & 4.5 & 10.2 & 50 \\
5 & 9 & 9 & 6.5 & 13.2 & 25 \\
6 & 12 & 6 & 6 & 13.2 & 25 \\
7 & 12 & 6 & 6 & 13.2 & 50 \\
8 & 12 & 9 & 6 & 13.2 & 25 \\
9
\end{tabular}

Table 8

Material properties for the drawbead test

\begin{tabular}{ll}
\hline Young's Modulus & $E=71000$ \\
Poisson's ratio & $v=0.34$ \\
Hardening behavior & $\sigma_{\mathrm{v}}=139(1+127.32852 \bar{\varepsilon} \mathrm{p})^{0.28}$ \\
\hline
\end{tabular}

curve. This means that, in our implementation, for a given precision, the Augmented Lagrangian algorithm is always cheaper than the Penalty one. These figures also show that, if high precision is required this can only be achieved by the Augmented Lagrangian algorithm.

\subsection{Blankholder with drawbead test}

Here we will deal with simulating the drawbead shown in Fig. 23. Different characteristic dimensions were investigated. The whole set of geometric parameters is given in Table 7 . The finite element mesh consists of 2 layers of 300 initially identical elements. The material is supposed to behave as an elastoplastic material with nonlinear isotropic hardening as described in Table 8. During the locking phase, the lower (male) part of the blankholder moves up until the given clamping force is reached. The pulling 


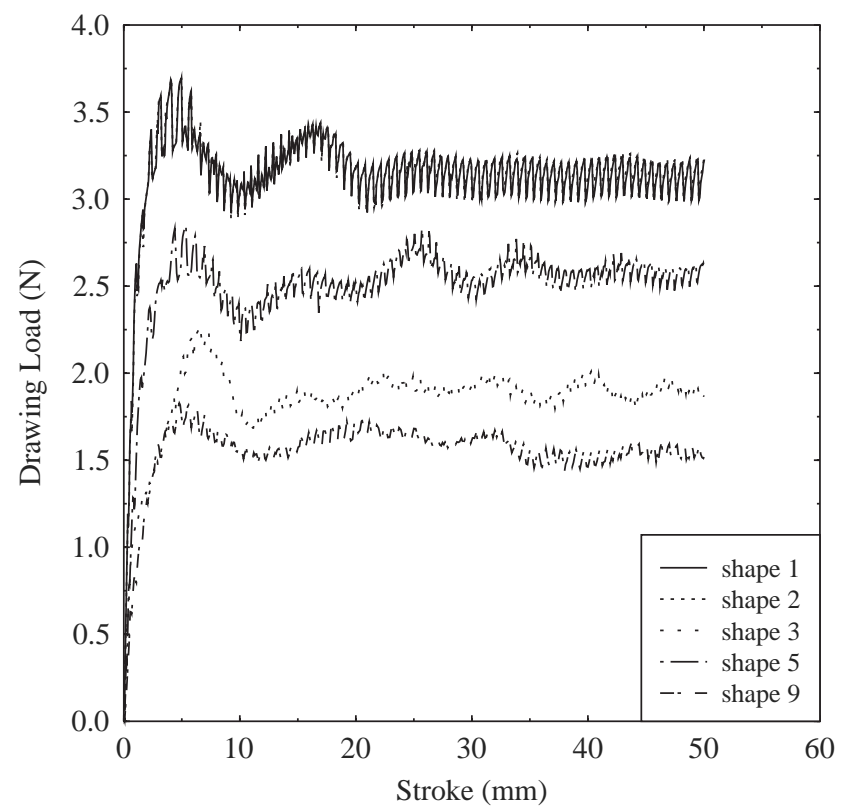

Fig. 24. Blankholder with drawbead, clamping.

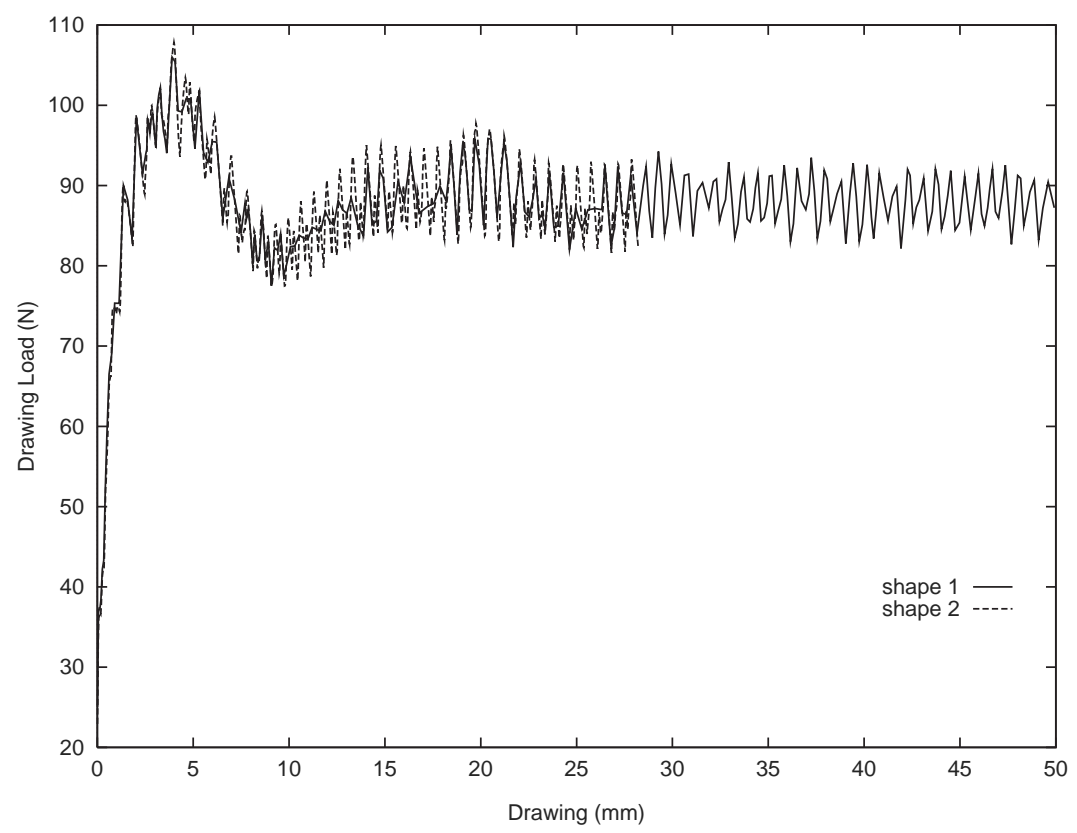

Fig. 25. Blankholder with drawbead, drawing.

phase which follows is characterized by a tangential displacement imposed on the right-hand side of the sheet, causing the sheet to bend and unbend as it is pulled through the bead. Our aim in this study is to analyse the effects of the parameters given in Table 7 on the restraining forces. The restraining contact 


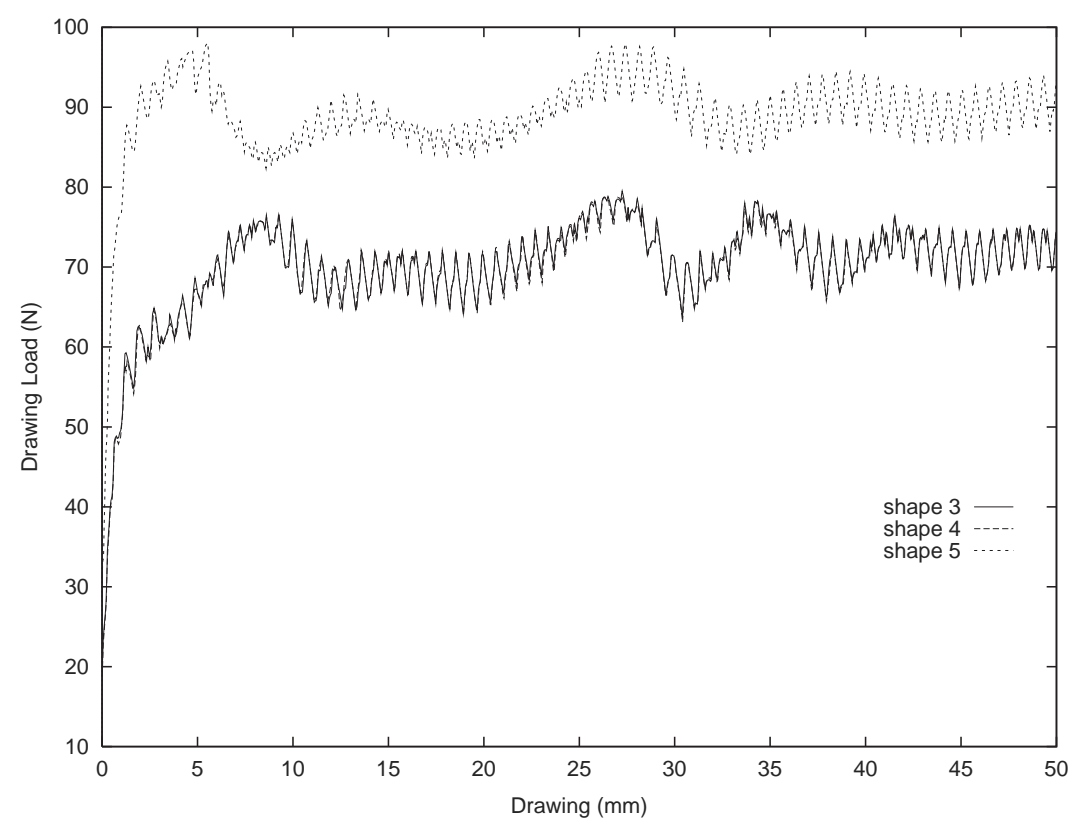

Fig. 26. Blankholder with drawbead, drawing.

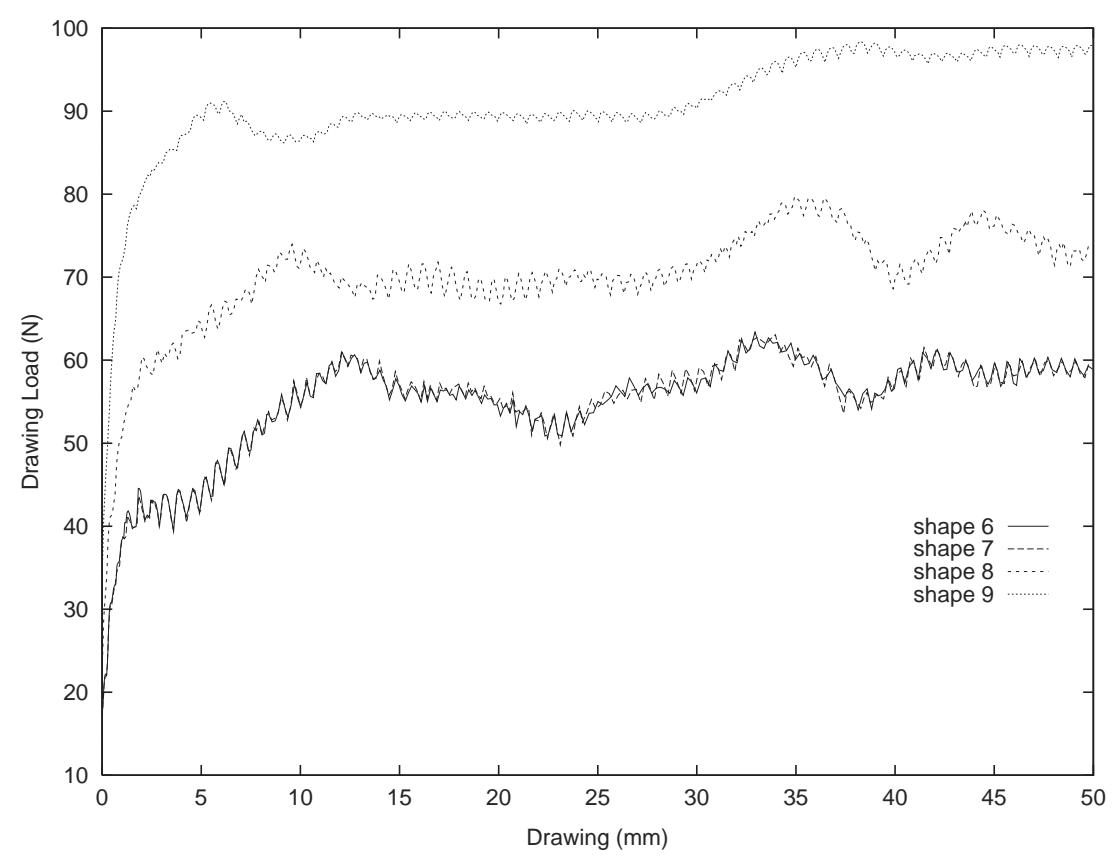

Fig. 27. Blankholder with drawbead, drawing. 
Table 9

Changes in the restraining forces with the various tool geometries

\begin{tabular}{llll}
\hline$R$ & $H$ & $L$ & Forces \\
\hline$\nearrow$ & Constant & Constant & $\searrow$ \\
Constant & $\nearrow$ & Constant & $\nearrow$ \\
$\nearrow$ & $\nearrow$ & Constant & $\searrow$ \\
Constant & Constant & $\nearrow$ & Constant \\
\hline
\end{tabular}

forces corresponding to the geometries of Table 7 are plotted in Fig. 24 in the case of the loading and in Figs. 25-27 in the case of the drawing. Shapes 1, 2 and 5 lead to the highest restraining forces. With constants $R$ and $H$, the variations in $L$ do not affect the drawing process. Table 9 summarizes the changes in the forces depending on the tool geometry.

\section{Conclusion}

The two finite element codes developed at Marseille and Liège constitute useful numerical tools for analyzing tribological devices. These tools when associated with experimental analysis, can help to reach a better understanding of the mechanisms involved in friction. Some phenomena which cannot be grasped by performing experimental measurements, such as local contact state, can actually now be modeled using a numerical simulation approach. In further studies, we intend to provide a more detailed analysis of the results obtained with each of these tests, including a larger number of parameters the evolution of which affects the restraining forces.

\section{References}

[1] B.D. Carleer, R. ter Haar, H. Huetink, D.J. Schipper, Sheet metal forming simulations with a friction model based on local contact conditions, in: J.K. Lee, G.L. Kinzel, R.H. Wagoner (Eds.), Numisheet'96, The Ohio State University, Dearborn, Michigan, 1996, pp. 40-46.

[2] P. Chabrand, O. Chertier, Variable friction coefficient model in deep drawing, in: T. Atlan (Ed.), Advanced Technology of Plasticity 1996, vol. 2, Colombus, Ohio, 1996, pp. 857-860.

[3] P. Chabrand, O. Chertier, Prise en compte du frottement sur les petits rayons: comparaison numérique-expérimental. Loi de frottement à coefficient variable, Tech. rep., Contrat CNRS/Regienov CNRS 119 affectation H5.12.51, 1995.

[4] P. Chabrand, F. Dubois, J.C. Gelin, Modelling drawbeads in sheet metal forming, Int. J. Mech. Sci. 38 (1) (1996) $59-77$.

[5] F. Dubois, Contact, frottement, grandes déformations élastoplastiques, Application à l'emboutissage, Ph.D. Thesis, Université de la Méditerranée, Marseille, 1994.

[6] D. Graillet, Modélisation tridimensionnelle des structure à parois minces pour des problèmes d'impact et de mise en forme, Ph.D. Thesis, University of Liège, Belgium, 2005, in course.

[7] H.D. Nine, New drawbead concepts for sheet metal forming, J. Appl. Metal Working 2 (3) (1982) $185-192$.

[8] H.D. Nine, The applicability of coulomb's friction law to drawbeads in sheet metal forming, J. Appl. Metal Working 2 (3) (1982) 200-210.

[9] H.D. Nine, Drawbeads forces in sheet metal forming, in: D. Kolstinen, N. Wang (Eds.), Mechanics of Sheet Metal Forming, Plenum Press, 1978, pp. 179-211.

[10] P. Chabrand, O. Chertier, F. Dubois, Complementarity methods for multibody friction contact problems in finite deformations, Int. J. Numerical Methods Eng. 51 (5) (2001) 553-578. 
[11] P. Chabrand, F. Dubois, M. Raous, Various numerical methods for solving unilateral contact problems with friction, Math. Comp. Modelling 28 (4-8) (1998) 97-108.

[12] A. Klarbring, G. Bjorkman, A mathematical programming approach to contact problem with friction and varying contact surface, Comp. Struct. 30 (1988) 1185-1198.

[13] E.H. Lee, Elastic-plastic deformation at finite strains, J. Appl. Mech. 36 (1969) 1-6.

[14] F. Sidoroff, Incremental constitutive equation for large strain elastoplasticity, Int. J. Eng. Sci. 20 (1) (1983) 19-26.

[15] J.C. Simo, R.L. Taylor, Consistent tangent operators for rate independent elastoplasticity, Comp. Meth. Appl. Mech. Eng. 48 (1985) 101-118.

[16] J.-P. Ponthot, A unified stress update algorithms for the numerical simulation of large deformations elastoplastic and elastoviscoplastic processes, Int. J. Plasticity 18 (2002) 91-126.

[17] J.-P. Ponthot, Traitement unifié de la mécanique des milieux continus solides en grandes transformations par la méthode des éléments finis, Ph.D. Thesis, University of Liège, Belgium, 1995.

[18] T. Laursen, Computational Contact and Impact Mechanics, Fundamental of Modeling Interfacial Phenomena in Nonlinear Finite Element Analysis, Springer, Berlin, 2002.

[19] T.A. Laursen, Formulation and treatment of frictional contact problems using finite elements, Ph.D. Thesis, Stanford University, 1992.

[20] T.A. Laursen, J.C. Simo, A continuum-based finite element formulation for the implicit solution of multibody, large deformation frictional contact problems, Int. J. Num. Meth. Eng. 36 (1994) 3451-3485. 